\title{
INFLUENNCIA DO CAVALO SOBRE A FERTILIDADE DO POLEN NO CAVALEIRO, EM CITRUS (*)
}

\author{
F. G. B r i e g e $r$ \\ e \\ J. T. A. Gurgel (**)
}

\section{A) - INTRODUÇ $\tilde{A} O$}

A influência do cavalo sobre o cavaleiro em enxertia é um problema que há muito tempo se discutiu, e uma interação entre cavalo e cavaleiro pode ser aceita hoje como fato comprovado. Para demonstrar essa relação tambem em formas de Citrur cultivadas em nosso país, foram iniciadas experiências em Piracicaba, Limeira e Ribeirão Preto $(1,18)$.

Estando a estudar, desde algum tempo, a fertilidade do polen em várias espécies e formas do gênero Citrus (19), constatamos que este era um carater facilmente determinavel. Ocorreu-nos, então, a idéia de usá-lo para demonstrar se existe quantitativamente uma influência do cavalo sobre a fertilidade do cavaleiro. Se isso fosse verificado, com muito mais razão poderíamos supor relações ainda mais pronunciadas entre cavalo e cavaleiro, em outras direções.

Desejamos, outrossim, consignar aquí os nossos agradecimentos aos srs. Filipe W. de Vasconcelos e Sílvio Moreira, pela permissão que nos deram de usar o material das suas experiências extensivas, de Piracicaba e Limeira.

(") Os estudos aquí apresentados fazem parte da análise pormenorizada de uma experiência sobre a influência de cavalo e cavaleiro para Citrus, em andamento na Estação Experimental de Limeira (18); posteriormente foi incluida uma experiência, tambem sobre o mesmo assunto, efetuada em Piracicaba (1).

(") Da Cadeira de Genética da Escola Superior de Agricultura "Luiz de Queiroz" da Universidade de São Paulo, Piracicaba. 


\section{B) - HISTÓRICO DO PROBLEMA "INTERAÇÃO ENTRE CAVALO E CAVALEIRO".}

Uma descrição, em minúcias, da literatura não será aquí apresentada, pois o nosso fim é mostrar apenas as etapas por que passou este interessante problema e o estado em que ele se acha atualmente.

De há muito, são relatados na literatura casos atribuidos à influência do cavalo ou porta-enxertos sobre o cavaleiro ou enxerto, quase sempre sob a forma de incompatibilidade ou não-ligação entre as partes, que se manifestam pelo crescimento exagerado do calo cicatricial, ou ainda pelo engrossamento desuniforme do cavalo ou cavaleiro, etc.. Com menos base, eram tambem citadas mudanças de gosto, forma, coloração etc., dos frutos do cavaleiro, influenciadas pelo cavalo.

Contudo, até há pouco se duvidava se de-fato a influência do cavalo sobre o cavaleiro ia alem dos efeitos causados pelo grau de ligação entre os tecidos do cavalo e cavaleiro na região da enxertia, dependendo disso os resultados mais ou menos satisfatórios da alimentação e do fornecimento suficiente de água pela raiz do cavalo d̀ copa do cavaleiro (l a 25).

Trabalhos mais modernos, porem, não deixam dúvidas de que, alem dos efeitos acima enumerados, há ainda os efeitos específicos entre as duas componentes ligadas artificialmente pela enxertia. Sem entrar em pormenores, podemos aquí referir-nos aos vários trabalhos de Hatton $(10,11,12)$ sobre a variação do tamanho da copa, do ângulo dos galhos, vigor das plantas, florescimento, produção, etc., em macieira e pereira, quando enxertadas sobre diferentes linhas (clones) de marmelo.

Igualmente, podemo-nos referir aos casos de metaxenia, em que uma interação semelhante à da enxertia se realiza na vida normal da planta - uma ligação íntima entre sementes com embriões híbridos, dentro de um fruto com tecido de origem puramente maternal. Nebel (20) demonstra que há, de-fato, tal interação em maçã e outros frutos cultivados na América-do-Norte, alterando-se a cor, gosto, etc., dos frutos, que são quase exclusivamente formados por tecido maternal, em dependência das sementes híbridas neles contidas. Observações semelhantes existem sobre laranja. De acordo com opinião de práticos, aumenta-se a acidez da laranja doce, em virtude da polinização e consequente formação de sementes, por fertilização com variedades azedas. Sobre este ponto, estamos fazendo estudos experimentalmente controlados.

Reportamo-nos ainda aos estudos anatômicos de quimeras, realizados por Haberlandt (5 a 8), que achou, para as quimeras periclinais de Crataegus e Méspilus, que a estrutura anatômica da epiderme de 
uma espécie é alterada pelos efeitos fisiológicos do tecido interno das hastes da outra espécie.

Em todos três casos citados (efeitos do cavalo sobre o cavaleiro, embrião híbrido sobre o tecido maternal do fruto, e componente sobre a outra em quimeras), temos de supor que são produzidas e distribuidas dentro das plantas substâncias específicas que podem ser chamadas de hormônios, e que produzem os referidos efeitos em tecidos mais ou menos distantes da sua origem (do hormônio).

O nosso trabalho está baseado num carater bem mais independente que os citados - o da fertilidade do polen do cavaleiro - o qual é rela. tivamente constante e típico para as espécies e variedades, como demonstramos noutra publicação (19). Podendo ser este carater tomado quantitativamente, é facil, pela análise estatística, notar e provar as diferenças importantes que apareçam.

\section{C) - MÉTODO DE DETERMINAÇÃO DO POLEN VIVO NAS LÂMINAS}

Os botões, em véspera de abrirem, eram colhidos com seus respectivos galhinhos, levados ao laboratório e conservados em frascos com água, até se dar a deiscêncía das anteras; este cuidado tornou-se indispensavel, a-fim-de evitar que o vento e principalmente as abelhas retirassem o polen das anteras. A seguir, preparávamos as lâminas e procedíamos à coloração com aceto-carmim.

A-fim-de que tivéssemos uniformidade nas determinações, estudávamos primeiramente a lâmina ao microscópio do "Panphoto", com bastante cuidado, excluindo assim a possibilidade de preferências por determinados campos; após esse exame preliminar, contávamos então 150 a 200 grãos de polen, correspondentes a 3 ou 5 diferentes campos.

Mais pormenores sobre o método usado se encontram em outro trabalho (19).

\section{D) - TESTE DE HOMOGENEIDADE DO POLEN VIVO}

Com o fim de obtermos base para a análise estatística nestas experiências de fertilidade do polen do cavaleiro em relação ao cavalo, fizemos preliminarmente um teste de homogeneidade entre as determinações do polen vivo.

Para esta análise, escolhemos o ensaio de cavalos para a laranja pera, existente na Estação Experimental de Limeira (ver capítulo F), 
escolhendo, ao acaso, 5 cavalos diferentes, e examinando, de cada um, 3 flores correspondentes a 3 árvores diferentes, de cada uma das 3 repetições, num total de 45 determinações (ou contagens). Foram contados 13800 grãos de polen, correspondendo à média de 230 por lâmina.

A seguir, foi feito um teste "Entre-Dentro", da percentagem do polen vivo, cujos resultados estão contidos no quadro $I$. $O \vartheta$ teste da última coluna mostra que nenhum erro difere significantemente do erro residual, donde concluirmos o seguinte : o erro entre-planta dentro-cavalo, diferindo insignificantemente do erro residual, mostra que as flores ou plantas foram escolhidas plenamente ao acaso; igualmente, sendo o erro entre 5 cavalos insignificantemente maior que o erro residual, indica que a variação é homogênea e inerente à própria experiência, e, porisso, inevitavel.

\section{QUADRO I}

ANÁLISE DA HOMOGENEIDADE DO MÉTODO

\begin{tabular}{|c|c|c|c|c|}
\hline \multicolumn{2}{|c|}{ Decomposição do Erro Total } & \multirow{2}{*}{$\begin{array}{l}\mathrm{nf} \\
44\end{array}$} & & $\vartheta=\frac{\sigma}{\sigma R}$ \\
\hline Total & $\sigma \mathrm{T}$ & & $\pm 3,94 \%$ & 0,97 \\
\hline Entre 5 cavalos & & 4 & $\pm 5,04 \%$ & 1,24 \\
\hline $\begin{array}{l}\text { Entre-planta } \\
\text { Dentro-cavalo }\end{array}$ & & 10 & $\pm 2,95 \%$ & 0,72 \\
\hline $\begin{array}{l}\text { Resíduo: } \\
\text { Dentro-planta } \\
\text { Entre-determinação }\end{array}$ & $\sigma R$ & 30 & $\pm 4,07 \%$ & 一 \\
\hline
\end{tabular}

Para que tivéssemos ainda uma idéia mais exata da homogeneidade da variação, fizemos a determinação do erro do polen vivo por árvores e por cavalo, e, ao mesmo tempo, estabelecemos até que limite esses erros podem variar (quadro II) ; como vemos, nenhum dos erros parciais excedeu $\circ$ limite de $1 \%$ de probabilidade, podendo-se, dessa forma, considerar a variação do polen como sendo bem homogênea.

Fazendo a determinação da média do polen vivo por árvore e por cavalo, estabelecemos os limites de variação das médias parciais (quadro 
III) ; aquí tambem não houve variação excessiva, distribuindo-se todas as médias parciais ao acaso, ao redor da média geral de 40,19\%, como se esperava, de acordo com o resultado do $\vartheta$ teste (quadro I).

A-final, para provar que a variação da percentagem do polen vivo encontrada é unicamente um resultado de fatores devidos ao acaso, comparamos pelo $\vartheta$ teste $\circ$ erro residual, obtido na experiência, com $\circ$ erro ideal, de uma distribuição binomial, calculado pela fórmula :

$$
\sigma i= \pm \sqrt{\frac{\overline{\overline{\mathrm{v}}} \%(100-\overline{\overline{\mathrm{v}}} \%)}{\mathrm{n}}}
$$

onde $\mathbf{n}$ é o número médio de grãos de polen por amostra, e $\overline{\bar{v}} \%$ a percentagem do polen vivo observado (determinado). Fazendo o quociente,

$$
\vartheta=\frac{\sigma \mathrm{R}}{\sigma \mathrm{i}}=\frac{4,07}{3,23}=1,26 \quad \frac{\mathrm{nf}_{\mathbf{1}}=30}{\mathrm{nf}_{\mathbf{2}}=\infty}
$$

obtemos um valor de $\vartheta$ que está entre os limites de $5 \%$ e $1 \%$ de probabilidade, podendo ser aceito como insignificante.

A-pesar dos muitos fatorez incontrolaveis que poderiam ter afetado a variação do polen vivo, pudemos demonstrar, satisfatoriamente, que não havia variação excessiva.

Assim, chejámos à conclusão de que os métodos de colheita e análise do poìen, aquí seguidos, eram bastante homogêneos, e que podemos, sem nenhuma hesitação, aplicá-los ao estudo da fertilidade do polen do cavaleiro sobre diferentes cavalos.

\section{E) - POMAR DA ESCOLA SUPERIOR DE AGRICULTURA "LUIZ DE QUEIROZ"}

\section{I - Considerações preliminares :}

Esta expəriência foi feita no pomar da Escola Superior de Agricultura "Luiz de Queiroz", de Piracicaba, pelo prof. Filipe Westin Cabral de Vasconcelos, a-fim-de verificar o comportamento das laranjas Baía comum, coco e natal sobre cinco diferentes cavalos: lima da Pérsia, laranja azeda e serra-d'áqua, limões francês e rugoso.

A plantação foi feita em linhas de 8 plantas cada uma, para as 15 combinações dos 3 cavaleiros com os 5 cavalos, na ordem acima enumerada (Mapa de plantação 1).

Segundo os dados gentilmente fornecidos pelo prof. Filipe W. C. Vasconcelos, os cavalos forams semeados em maio de 1926, enxertados 


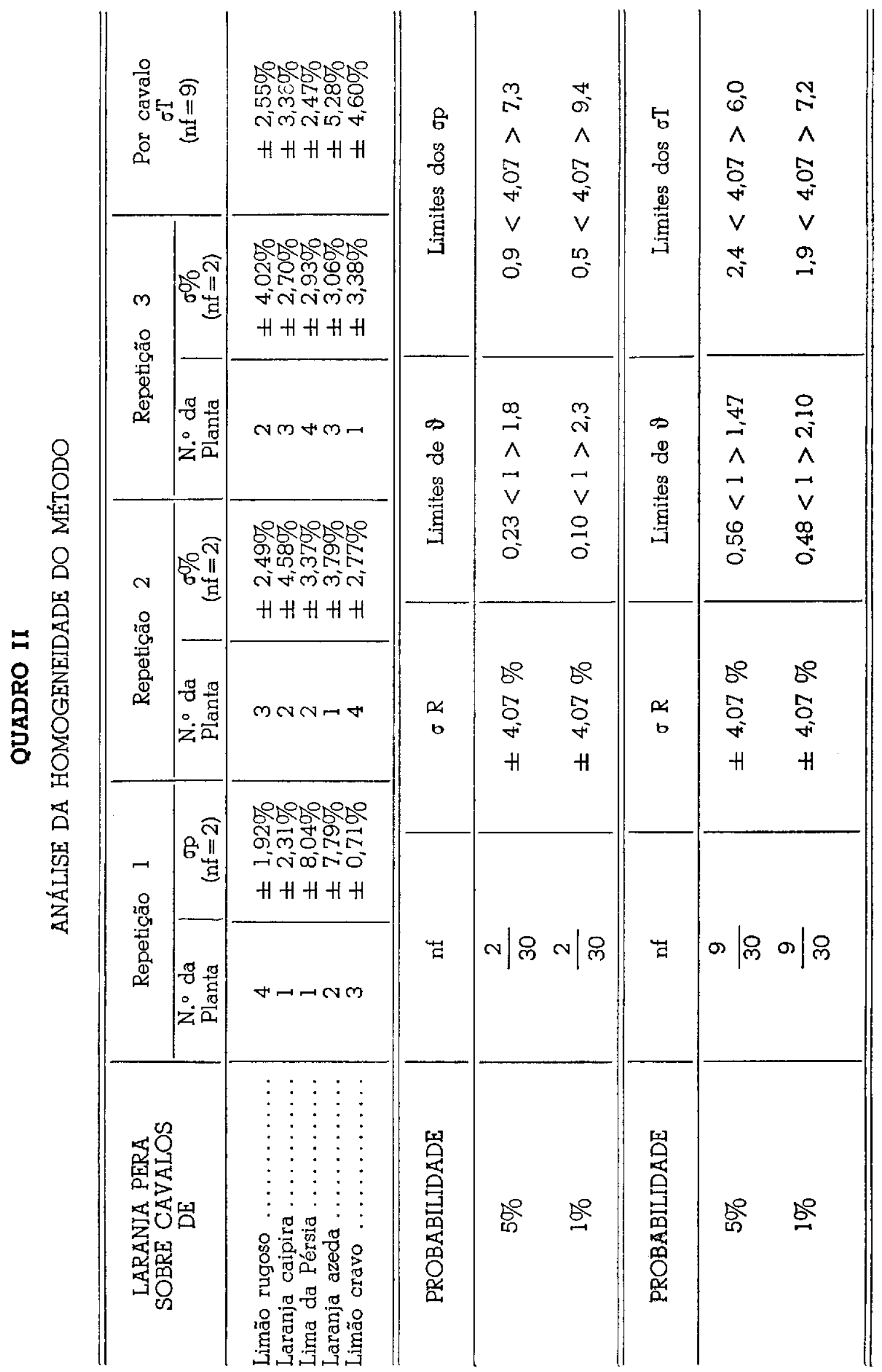




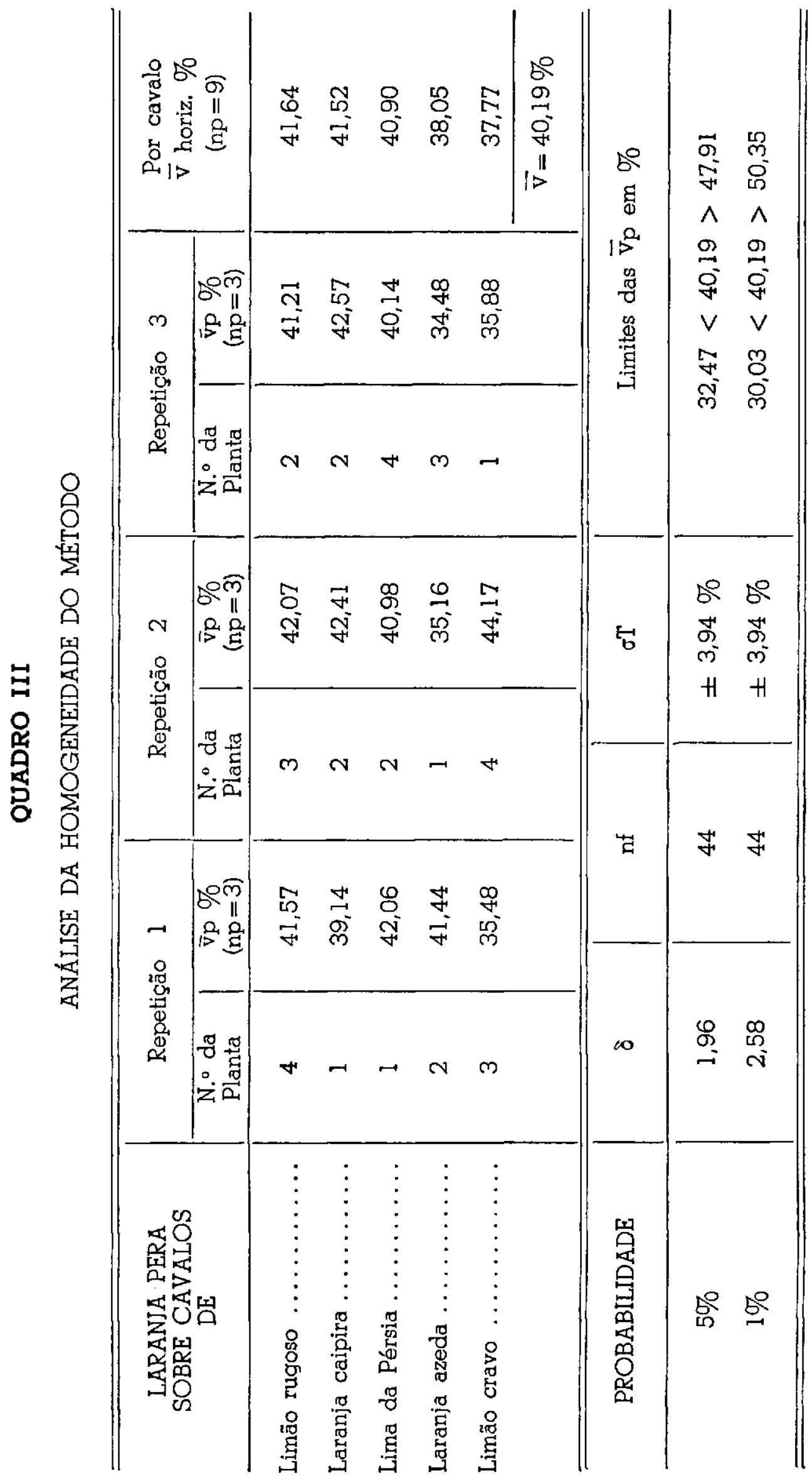


em outubro de 1928 e transplantados para o lugar definitivo em janeiro de 1930 (1).

As determinações da fertilidade do polen para os 3 cavaleiros foram feitas em setembro de 1939. Na falta de repetições de linhas (combinações diferentes), foram examinadas 3 flores de cada planta, o que facilitaria, na análise estatística, melhor apreciação do erro residual ou experimental.

\section{II - Método de análise estatística empregado :}

O erro total foi dividido em duas componentes : o erro entre-cavalo e o erro dentro-cavalo. Uma vez que tínhamos analisado 3 flores de cada planta, foi possivel subdividir o erro dentro em duas outras componentes: o erro entre-plantas/dentro-cavalos e o erro dentro-plantas/ entre-determinação. Isso talvez pudesse compensar a falta de repetições dos cavalos.

Todavia, como adiante veremos, essa subdivisão do erro residual foi de pouco valor, sendo melhor usar sempre o erro residual com o maior grau de liberdade.

\section{III - Análise da laranja natal :}

Para a análise do polen vivo de laranja natal enxertada sobre 5 diferentes cavalos, foram escolhidos de cada cavalo, ao acaso, 3 plantas, e de cada planta examinadas 3 flores; houve uma exceção com o cavalo de laranja azeda, em que encontramos, na ocasião do nosso trabalho, apenas duas plantas com flores. Temos, pois, para o conjunto, 14 plantas analisadas, num total de 42 determinações (Mapa de plantação 1). Nesta experiência foram contados 7644 grãos de polen, dando uma média de 182 por lâmina.

$O$ erro total da percentagem do polen vivo, conforme consta no quadro IV, foi dividido em três partes, a última das quais pode ser considerada como erro experimental (4. " linha do quadro). Dos dois valores de $\vartheta$, um foi duvidoso (aquele para o erro entre-cavalos), enquanto que o outro foi insignificante. Em consequência disso, podemos reunir as últimas componentes (entre-planta/dentro-planta) em um único erro residual (5. "linha do quadro), com nf $=39$. Fazendo mais uma vez o $\vartheta$ teste (última coluna do quadro IV), o erro entre-cavalos ainda fica duvidoso. 


\section{QUADRO IV}

\section{PIRACICABA - LARANJA NATAL SOBRE 5 CAVALOS}

\begin{tabular}{|c|c|c|c|c|c|}
\hline DECOMPOSIÇAO DO ERRO & OTAL & $\mathrm{nf}$ & $\sigma$ & $\vartheta=\frac{\sigma}{5.66 \%}$ & $\vartheta=\frac{\sigma}{5.48 \%}$ \\
\hline Total $\ldots \ldots \ldots \ldots \ldots \ldots$ & $\sigma \mathrm{T}$ & 43 & $\pm 6,06 \%$ & 1,07 & 1,10 \\
\hline Entre 5 cavalos......... & $\sigma \mathrm{E} 5 \mathrm{C}$ & 4 & $\pm 9,08 \%$ & $(1,60)$ & $(1,66)$ \\
\hline Dentro-cavalo & & & & & \\
\hline $\begin{array}{l}\text { Entre-planta } \\
\text { Dentro-cavalo }\end{array}$ & & 10 & $\pm 6,16 \%$ & 1,09 & 1,12 \\
\hline $\begin{array}{l}\text { Dentro-planta } \\
\text { Entre-determinação }\end{array}$ & & 29 & $\pm 5,48 \%$ & 0,97 & - \\
\hline $\begin{array}{l}\text { Resíduo } \\
\text { Dentro-cavalo ........... }\end{array}$ & $\sigma R$ & 39 & $\pm 5,66 \%$ & - & 1,03 \\
\hline
\end{tabular}

Para termos uma idéia mais segura sobre o que indica esse $\vartheta$ duvidoso, calculamos as médias parciais $\overrightarrow{\mathrm{v}} \mathrm{p}$ para cada cavalo e determinamos os limites da variação ao acaso destas $\overline{\mathrm{v}} \mathrm{p}$ ao redor da média geral ; isto deverá mostrar se existe, ou não, algum cavalo cujo cavaleiro tenha uma quantidade de polen vivo fora dos limites esperados. Como se depreende do quadro $\mathrm{V}$, nenhuma média parcial excedeu o limite de $5 \%$ de probabilidade, donde concluir-se que $\circ \vartheta$ duvidoso, para o erro entrecavalos, não tem importância, e que toda a variaçăo foi devida ao acaso.

Parece, pois, que nesta expəriência nenhum dos cavalos usados teve o menor efeito sobre a fertilidade do polen da laranja natal.

\section{IV - Análise da laranja coco :}

A or yanização e análise desta experiência são idênticas às da anterior, tendo-se aplicado a mesma técnica : 5 cavalos $\times 3$ plantas $\times 3$ flores $=45$ determinações (Mapa de plantação 1). Os grãos de polen contados atingem a 6518, dando a média de 145 por lâmina.

Decompondo em três partes o erro total da percentagem do polen vivo, podemos ver (quadro VI) que os dois erros (entre-planta e dentroplanta) são, mais uma vez, quase iguais, de modo que é aconselhavel reuní-los em um único erro residual, com o maior grau de liberdade. 


\section{QUADRO V}

PIRACICABA - LARANJA NATAL SOBRE 5 CAVALOS

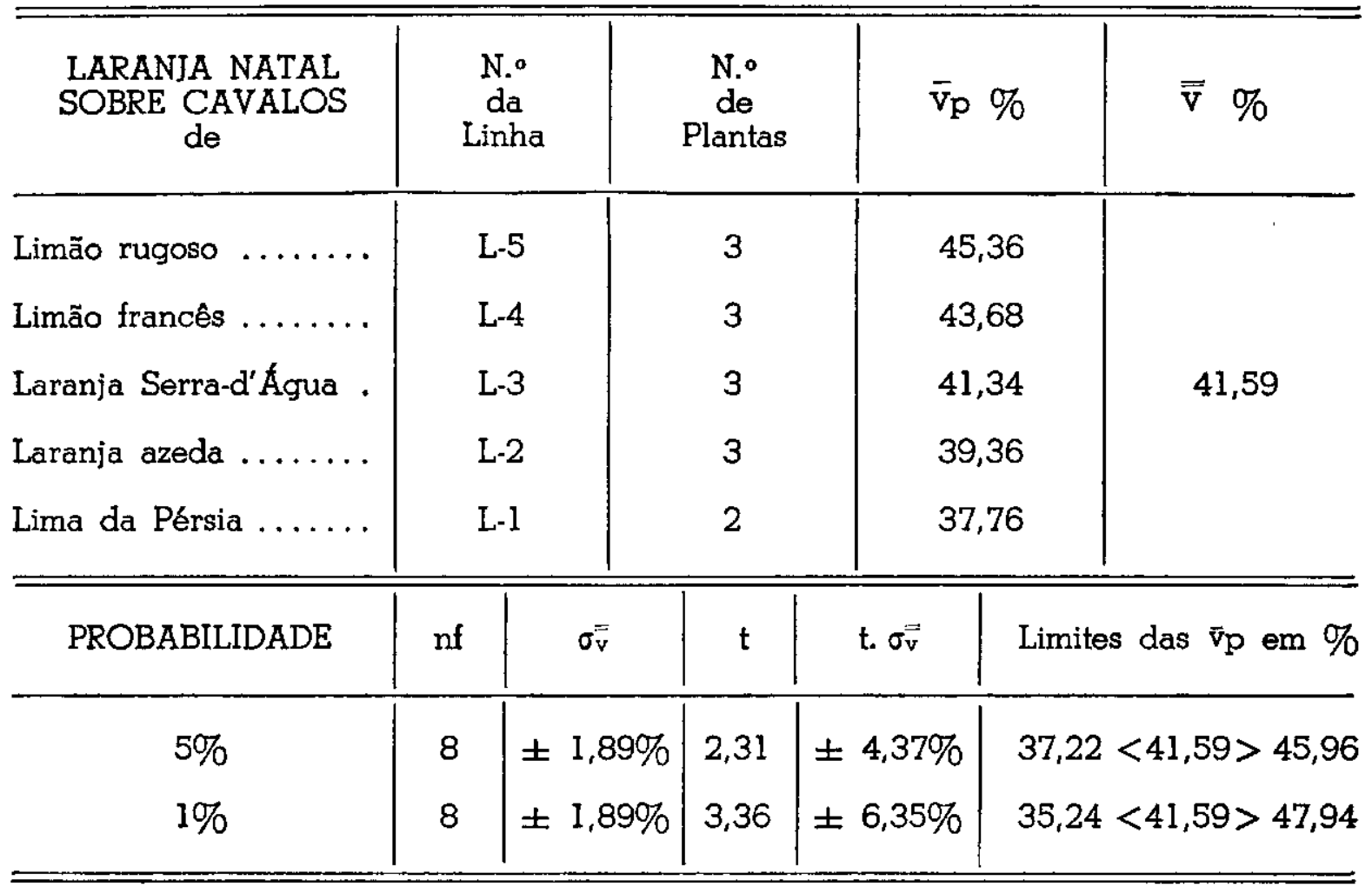

Usando, então, este valor do erro residual, obtivemos para o erro entrecavalos um valor de $\vartheta$ duvidoso.

Para acharmos a razão por que $\circ \vartheta$ do erro entre-cavalos foi duvidoso, determinamos os limites da variação das médias parciais ao redor da média geral (quadro VII) ; por este último teste, pudemos ver que existe um cavalo (laranja azeda) cuja média fica abaixo do limite de $5 \%$ de probabilidade.

Podemos assim tentar um agrupamento dos cavalos, sendo um grupo constituido só do cavalo de laranja azeda e outro dos 4 cavalos restantes: lima da Pérsia, laranja serra-d'água, limão francês e rugoso.

Este agrupamento nada mais é que uma nova decomposição do erro entre-cavalos (2. ${ }^{\text {a }}$ metade, à direita, do quadro VI). $\bigcirc \vartheta$ do erro entre dois grupos, dividido pelos dois diferentes erros residuais, sendo significante, mostra-nos que os dois grupos são de-fato diferentes. $\bigcirc \vartheta$ do erro dentro-grupo, dividido pelos dois erros residuais, sendo insignificante, indica-nos que o grupo de 4 cavalos é bem uniforme. 


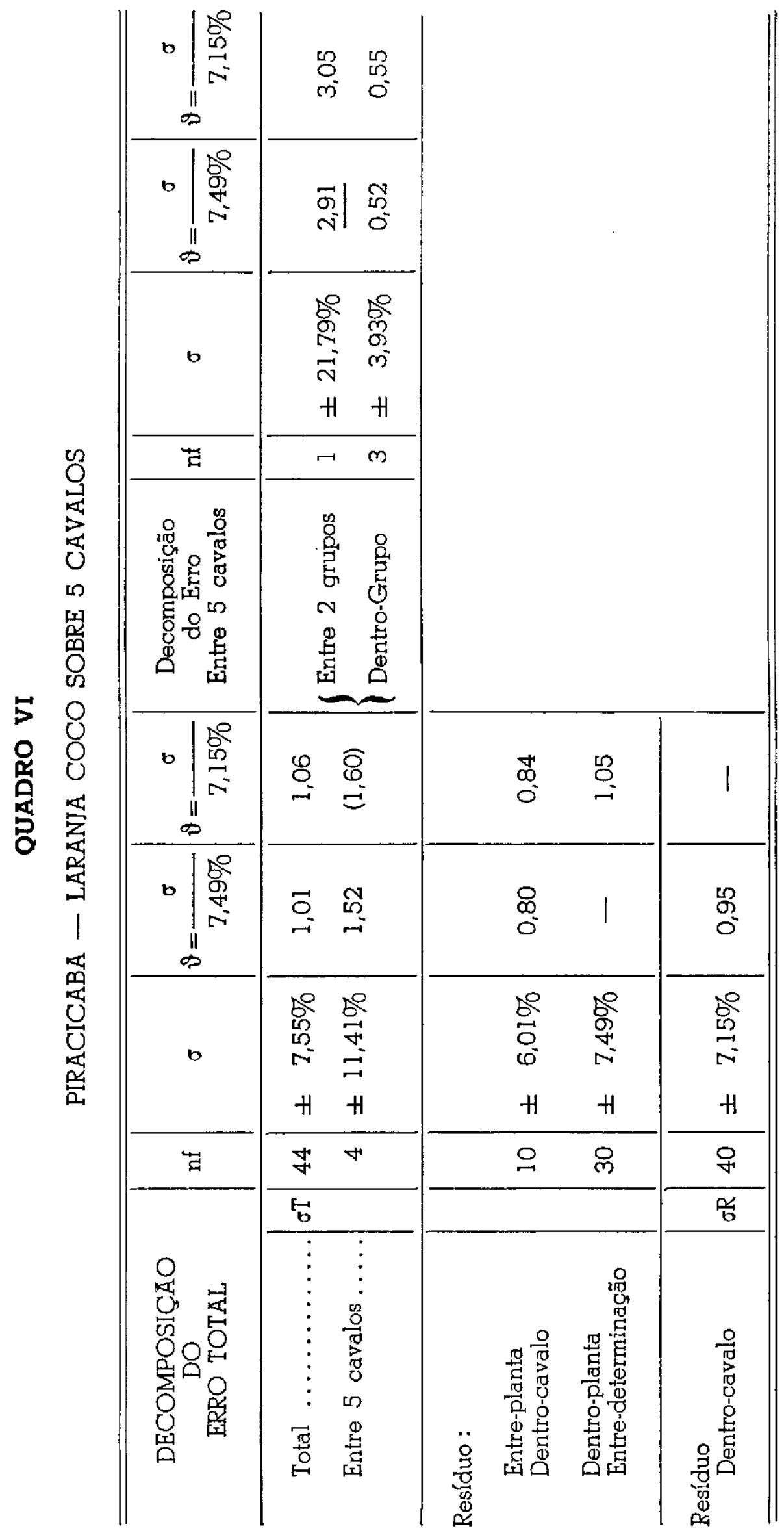




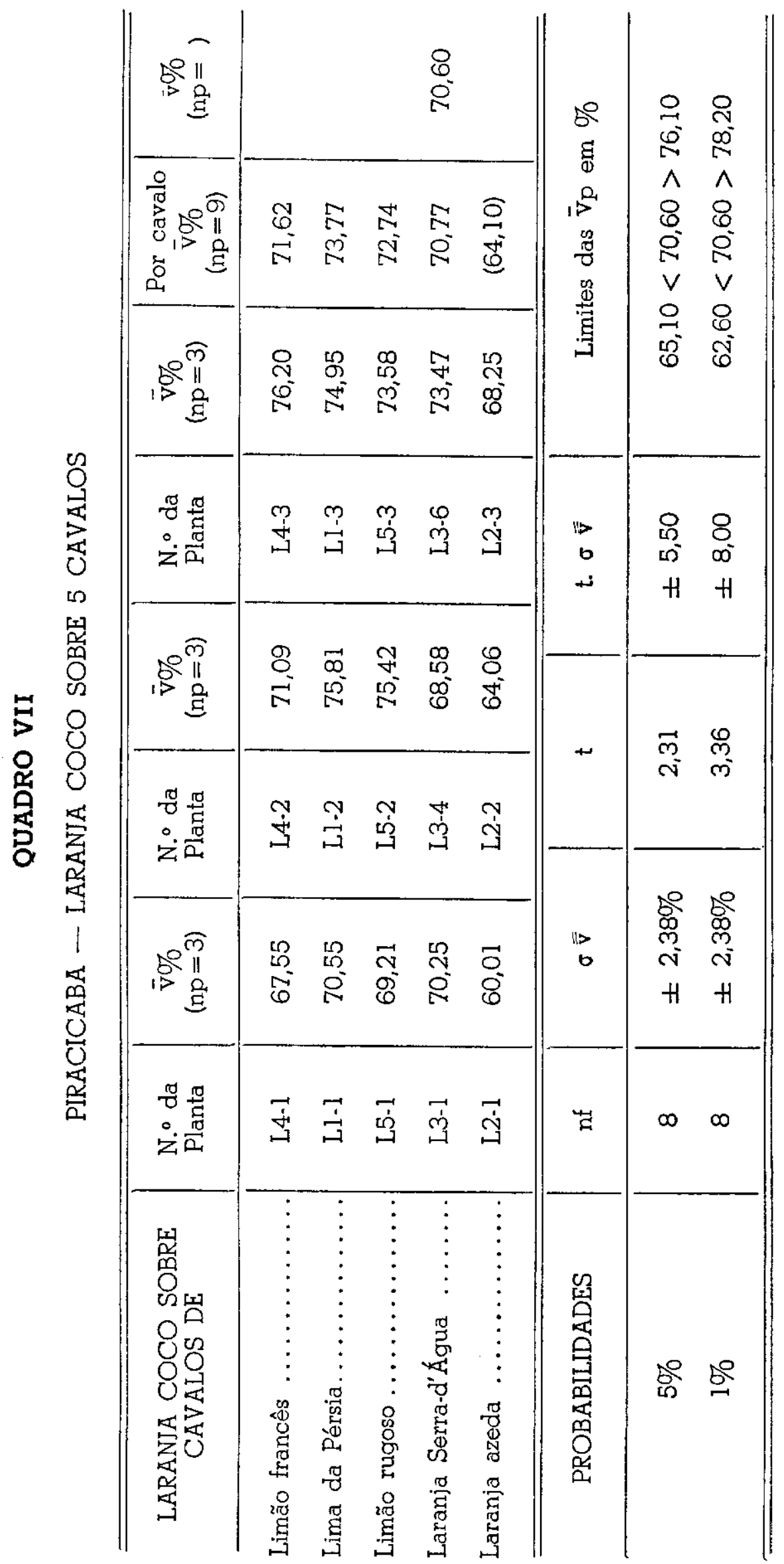




\section{V - Análise da laranja Baía comum :}

O exame ao microscópio do polen de laranja Baía comum sobre os 5 diferentes cavalos revelou uma esterilidade completa. Já esperávamos esse resultado, uma vez que, em todas as formas de laranja Baía, sempre encontramos a ausência de células vivas do polen (19).

\section{F) - ESTAÇÃO EXPERIMENTAL DE LIMEIRA - INSTITUTO AGRONÔMICO}

\section{I - Considerações preliminares :}

Neste ensaio está sendo estudado o comportamento das nossas principais variedades comerciais de Citrus, tais como a laranja baia. ninha Piracicaba, laranja pera e o pomelo "Marsh-seedless" sobre 12 diferentes cavalos. Estes foram divididos em dois grupos, correspondentes a duas quadras: A) constituido pelos cavalos de laranja azeda, azeda-doce e caipira, lima da Pérsia e limão cravo e rugoso; B) constituido pelos cavalos de tangerina cravo, laranja lima, cidra, limão pon. derosa, trifoliata e pomelo triunfo.

Para cada tipo de cavaleiro foi feito um ensaio, subdividido em duas quadras, uma para os cavalos do grupo $\mathrm{A}$ e outra para os do grupo B. Cada quadra foi ainda repartida em quatro blocos iguais, correspondentes a 4 repetições completas. Dentro de cada bloco, as parcelas foram distribuidas ao acaso.

$\mathrm{Na}$ quadra $\mathrm{A}$, as parcelas são formadas de 9 plantas; como foi repetida 4 vezes, temos 36 árvores por cavalo e cavaleiro. Comportando cada bloco 6 parcelas, correspondentes a 6 cavalos, temos então 24 parcelas e um total de 216 plantas.

$\mathrm{Na}$ quadra $\mathrm{B}$, as parcelas são formadas de 4 plantas; como foi repetida 4 vezes, temos 16 árvores por combinação de cavalo e cavaleiro. Comportando, igualmente, cada bloco 6 parcelas, correspondentes aos 6 cavalos, temos tambem 24 parcelas e um total de 96 plantas.

Finalmente, somando as duas quadras, teremos ao todo 312 plantas para cada ensaio (Mapas de plantação 2 e 3).

As áreas ocupadas pela experiência são:

Área em metros quadrados.

\section{Parcela Bloco}

Quadra A

Quadra B

576

256
3456

1536
Quadra

13824

6144

19968 
Os dados da experiência, gentilmente cedidos pelos sr. Sílvio Moreira (18), são: semeação dos cavalos em agosto de 1933, enxertia em fevereiro de 1935, transplantação para o pomar em dezembro de 1936. As borbulhas de uma mesma variedade de cavaleiro foram retiradas de uma só planta, evitando-se assim introduzir variações individuais.

As determinações do polen vivo foram feitas de outubro a novembro de 1940. Uma vez que havia repetições para as parcelas, o que permitia uma análise detalhada do erro residual, foi examinada apenas uma flor de cada árvore. Isso era justificavel, pois, como mostramos no teste de homogeneidade, não havia variação dentro das plantas.

\section{II - Método de análise estatística empregado :}

A análise estatística torna-se nesta experiência bem completa. Ao mesmo tempo, entretanto, apresenta-se mais complicada do que no ensaio anterior. A existência de quatro repetições completas permite agora uma avaliação do efeito do terreno, o que não foi possivel anteriormente.

$\mathrm{Na}$ experiência de Piracicaba, podíamos distinguir, na decomposição do erro total, duas componentes principais: o erro entre-cavalos e o erro dentro ou residual. Agora teremos, em vez disso, a componente erro entre-parcelas, que deverá, por seu turno, ser dividida em três partes: entre-cavalos, entre-repetições e a interação cavalo-repetição; a outra componente será, tambem aquí, o erro residual.

$O$ erro residual foi anteriormente subdividido em: entre-plantal dentro-cavalo e dentro-planta/entre-determinação; esta subdivisão, naquela experiência, parecia ser necessária, uma vez que não havia repetições e tínhamos examinado 3 flores por planta. Sendo, porem, esses dois erros iguais (quadro III $e \mathrm{~V}$ ), e havendo repetições no ensaio de Limeira, essa subdivisão do erro residual foi desprezada.

Uma outra complicação provem da divisão geográfica do ensaio em duas quadras. Não podendo esperar uniformidade entre elas, dada a grande área que ocupam, a análise tinha, em primeiro lugar, de tomá-las separadamente ; foi tentada, a-final, uma análise em conjunto, fundindo( $\left.{ }^{*}\right)$ os dados das duas quadras.

\section{III - Análise de laranja pera :}

Embora tencionássemos determinar o polen vivo do maior número possivel de plantas do ensaio, a fatigante contagem e o tempo relativamente curto da floração impossibilitaram-nos de fazê-lo; mesmo assim,

(*) Traduzimos aquí o termo técnico da literatura inglesa "confounding" pelo verbo "fundir". 
examinamos no mínimo 3 árvores de cada parcela, correspondentes aos 12 diferentes cavalos, nas 4 repetições e nas duas quadras, num total de 161 plantas (Mapa de plantação 2). Foram assim contados um total de 31073 grãos de polen, o que dá uma média de 192 por enxerto.

Fazendo a decomposição do erro total da percentagem do polen vivo (quadro VIII), podemos discutir o seguinte: a variação entre-repetições para as duas quadras é insignificante, quando comparada com o erro residual, de modo que consideraríamos o terreno como razoavel. mente uniforme. A variação entre-cavalos é muito significante na quadra $A$ e talvez significante na quadra $B\left(4 .{ }^{a}\right.$ e $8 .{ }^{a}$ colunas do quadro VIII). Nesta última, aparece ainda uma interação significante, indicando que os diferentes cavalos não se comportam de modo igual nas 4 repetições, ao passo que na quadra $A$ não houve interação.

Em consequência da grande extensão da área experimental, pareceunos necessário fazer um teste de homogeneidade. Comparamos, por isso, o erro dentro por cavalo $\left(\sigma D R^{\prime}\right)$ com o erro residual da análise da experiência completa, que tem o valor de $\pm 4,51 \%$, e é baseado em 118 graus de liberdade. Os respectivos valores de $\vartheta$ encontram-se na última coluna do quadro IX; de todos onze valores de $\vartheta$, dez são pequenos e insignificantes e apenas um é duvidoso. Estamos, portanto, justificados, admitindo a experiência como suficientemente homogênea.

Os erros residuais nas duas quadras são praticamente idênticos, sendo para A de $\pm 4,79 \%$ e para B de $\pm 4,19 \%$, e as componentes Entre tambem parecem ser da mesma dimensão (quadro VIII). Em consequência disso, pareceu-nos razoavel fundir os dados das duas quadras, e os resultados dessa fusão constam na última parte do quadro VIII.

Devido a essa fusão, aparece uma nova componente, a variação Entre quadras, a qual é, no caso em discussão, muito significante. Podemos tambem fazer um teste para a diferença das médias do polen vivo da quadra A, que é de $40,72 \%$ e baseada em 80 observações ; para a quadra B é de 44,63\%, baseada em 81 observações.

$$
\delta=\frac{\overline{\mathrm{v}}_{1}-\overline{\mathrm{v}}_{2}}{\sigma \overline{\mathrm{v}} \operatorname{dif}}=\frac{\overline{\mathrm{v}}_{1}-\overline{\mathrm{v}}_{2}}{\sigma \sqrt{\frac{1}{\mathrm{n}_{1}}+\frac{1}{\mathrm{n}_{2}}}}=\frac{44,63-40,72}{4,7 \mathrm{l} / \sqrt{\frac{1}{81}+\frac{1}{80}}}=+5,51 \quad \frac{\mathrm{nf}_{1}=1}{\mathrm{nf}_{2}=159}
$$

$\bigcirc$ valor $\delta$ de $+5,51$ é muito significante, permitindo-nos dizer que, do ponto de vista da estatística, as duas quadras são bem diferentes.

A variação do terreno dentro das quadras fica naturalmente insignificante. A interação significante mantem-se e a variação entre cavalos cresce ainda mais. 


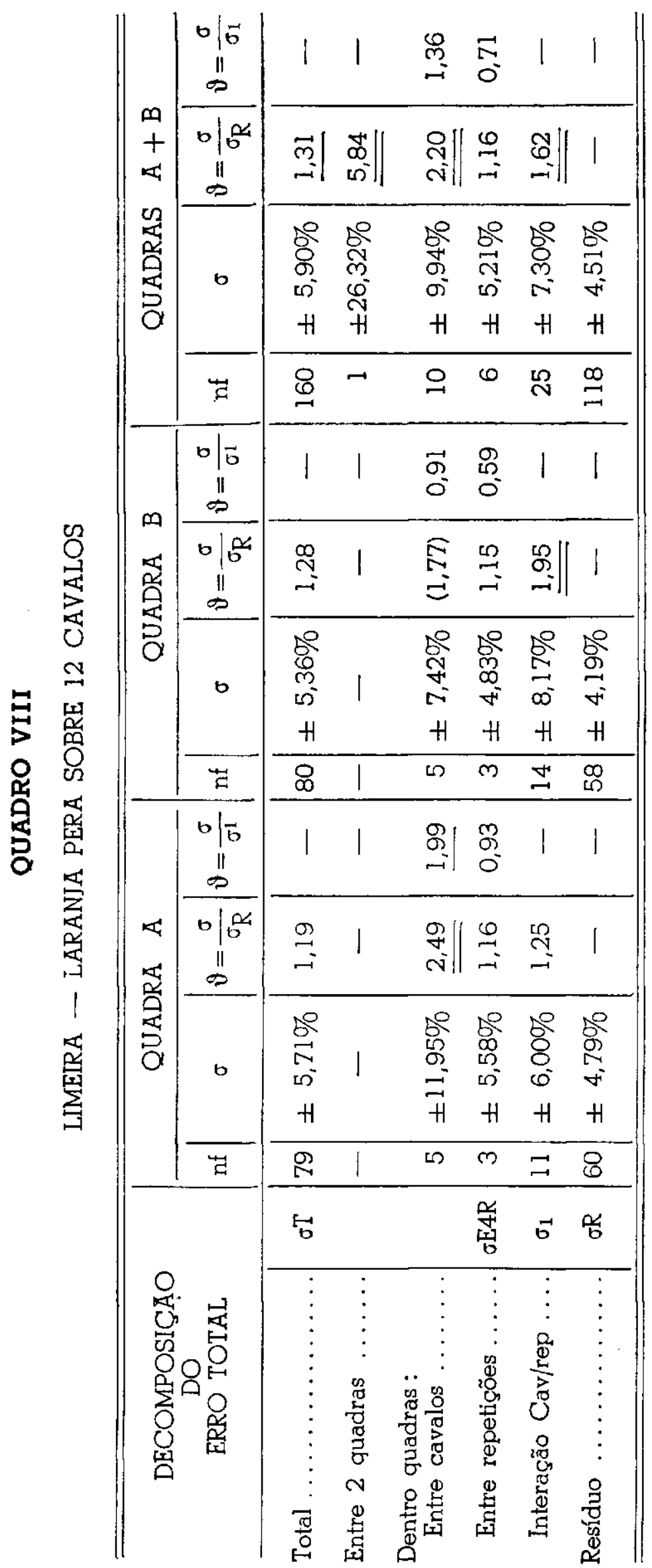




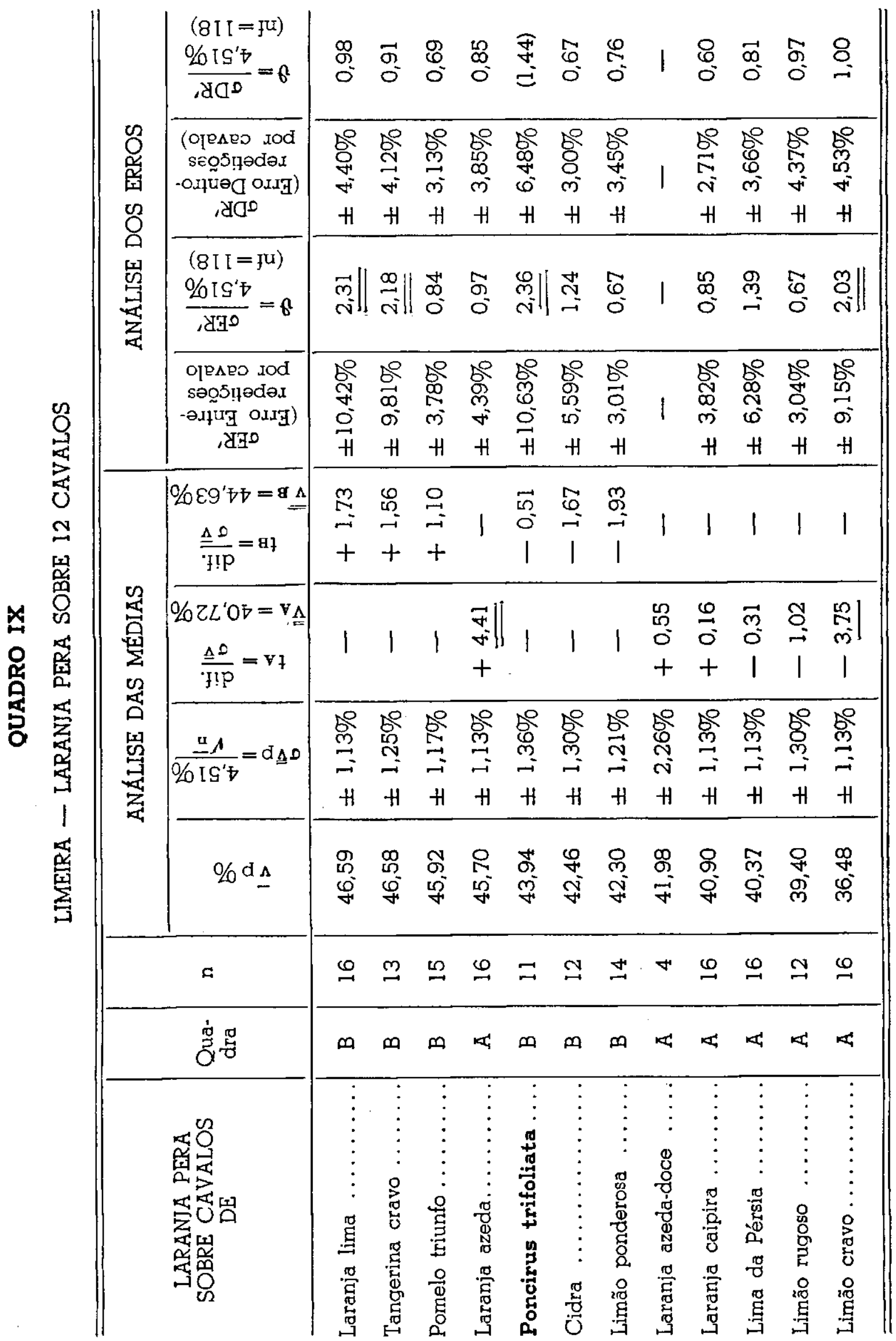


A causa da interação, vamos vê-la no quadro $X$, que contem as médias por cavalo e repetição. A variação não paralela dos diferentes cavalos, especialmente na quadra B, é bem evidente. Assim, a laranja lima tem o valor máximo na repetição 2 e o mínimo na repetição 1 ; a tangerina cravo, ao contrário, tem o máximo na repetição l e o mínimo na repetição 4 ; o pomelo triunfo tem o valor máximo na repetição 3 e o mínimo na repetição 1 , etc.

\section{QUADRO X}

\section{LIMEIRA - LARANJA PERA SOBRE 12 CAVALOS}

\begin{tabular}{|c|c|c|c|c|c|c|}
\hline $\begin{array}{l}\text { Grupo } \\
\text { ou } \\
\text { Quadra }\end{array}$ & $\begin{array}{c}\text { LARANJA PERA } \\
\text { SOBRE CAVALOS } \\
\text { DE }\end{array}$ & $\begin{array}{l}\text { Repet } \\
1 \\
-\mathrm{vp} \%\end{array}$ & $\begin{array}{l}\text { Repet } \\
2 \\
-\mathrm{vp} \%\end{array}$ & $\begin{array}{l}\text { Repet } \\
3 \\
\mathrm{vp} \%\end{array}$ & $\begin{array}{l}\text { Repet } \\
\quad 4 \\
\text { vp } \%\end{array}$ & $\begin{array}{c}\overrightarrow{\mathrm{v}} \\
\text { horiz. } \\
\%\end{array}$ \\
\hline \multirow[t]{2}{*}{$A$} & $\begin{array}{l}\text { Laranja azeda } \ldots \ldots \ldots \ldots \\
\text { Laranja azeda-doce } \ldots \ldots \ldots \\
\text { Laranja caipira } \ldots \ldots \ldots \ldots \\
\text { Lima da Pérsia } \ldots \ldots \ldots \ldots \\
\text { Limão rugoso } \ldots \ldots \ldots \ldots \ldots \\
\text { Limão cravo } \ldots \ldots \ldots \ldots \ldots\end{array}$ & $\begin{array}{l}42,62 \\
41,98 \\
41,02 \\
44,94 \\
39,67 \\
38,11\end{array}$ & $\begin{array}{l}47,82 \\
43,24 \\
37,98 \\
37,76 \\
29,66\end{array}$ & $\begin{array}{l}46,31 \\
40,78 \\
38,71 \\
40,76 \\
38,87\end{array}$ & $\begin{array}{l}46,03 \\
- \\
38,56 \\
39,84 \\
39,28\end{array}$ & $\begin{array}{l}45,70 \\
41,98 \\
40,90 \\
40,37 \\
39,40 \\
36,48\end{array}$ \\
\hline & $\overline{\mathrm{v}}$ vert. $\%$ & 41,39 & 39,29 & 41,09 & 40,93 & 40,72 \\
\hline \multirow[t]{2}{*}{ B } & $\begin{array}{l}\text { Laranja lima } \ldots \ldots \ldots \ldots \\
\text { Tangerina cravo } \ldots \ldots \ldots \ldots \\
\text { Pomelo triunfo } \ldots \ldots \ldots \ldots \\
\text { Poncirus trifoliata } \ldots \ldots \ldots \\
\text { Cidra } \ldots \ldots \ldots \ldots \ldots \\
\text { Limão ponderosa } \ldots \ldots \ldots \ldots\end{array}$ & $\begin{array}{l}41,81 \\
54,07 \\
43,62 \\
50,24 \\
47,04 \\
43,02\end{array}$ & $\begin{array}{l}53,08 \\
42,62 \\
44,89 \\
40,54 \\
42,07 \\
40,53\end{array}$ & $\begin{array}{l}43,00 \\
47,80 \\
47,90 \\
- \\
39,45 \\
41,35\end{array}$ & $\begin{array}{l}48,46 \\
41,84 \\
47,28 \\
41,05 \\
41,26 \\
44,30\end{array}$ & $\begin{array}{l}46,59 \\
46,58 \\
45,92 \\
43,94 \\
42,46 \\
42,30\end{array}$ \\
\hline & $\overrightarrow{\mathrm{v}}$ vert. $\%$ & 46,63 & 43,96 & 43,90 & 44,03 & 44,63 \\
\hline uadras $(A+B)$ & $\overline{\mathrm{v}}$ vert. $\%$ & 44,01 & 41,84 & 42,49 & 42,79 & 47,68 \\
\hline
\end{tabular}

mesmo está demonstrado pela comparação dos erros entre-repetições por cavalo $\left(\sigma E R^{\prime}\right)$ com o erro residual da experiência (coluna 9, quadro IX) : nesta comparação foi usado, como base, o erro residual, uma vez que o erro entre-repetições não foi diferente dele (quadro VIII). Podemos notar que há um grupo bastante heterogêneo de cavalos ( 3 cavalos da quadra $A$ e 1 da quadra $B$ ), os quais se comportam diferentemente nas repetições; apenas 7 dos $\left(\sigma E R^{\prime}\right)$ diferem insignificantemente do erro residual da experiência, formando um grupo bem homogêneo. Podemos assim tentar a reunião dos $\sigma E R^{\prime}$ em dois grupos: um com $4 \sigma E R^{\prime}$ variando de 9 a $10 \%$ e outro com $7 \sigma E R^{\prime}$ variando de 3 a $6 \%$. Para o 
primeiro grupo temos um erro balançado entre-repetições por cavalo de $\pm 9,60 \%$, com $\mathrm{nf}=11$ e para o segundo grupo o erro balançado de $\pm 4,48 \%$, com nf $=20$. Fazendo um $\vartheta$ teste do segundo com $\circ$ erro residual, vemos que é insignificantemente menor, indicando que para este último o terreno foi uniforme.

A variação não paralela dos cavalos nas repetições e a desigualdade dos erros entre-repetições por cavalo talvez expliquem o aparecimento da interação, pois, evidentemente, há manchas grandes, do tamanho da parcela $\left(576 \mathrm{~m}^{2}\right)$, e irregularmente distribuidas no terreno; os sistemas radiculares dos diferentes cavalos talvez possam responder diferentemente aos efeitos edáficos.

O ponto principal, porem, é a análise da variação circunstanciada dos erros entre-cavalos. $\mathrm{Na} 4 .^{\text {a }}$ coluna do quadro IX encontramos os valores das médias parciais $\overline{\mathrm{v}} \mathrm{p}$ por cavalo e os respectivos erros das médias ō̃p; na 6. e 7.a colunas se acham os valores de $t$ para as diferenças entre as médias $\bar{v}$ p dos cavalos com a média geral $\bar{v}$ de cada quadra. Os cavalos estão organizados em ordem decrescente das médias parciais.

Em primeiro lugar notamos que os enxertos da quadra $A$ teem, em geral, menos polen fertil que os da quadra B.

Comparando os 6 cavalos da quadra B com a sua média geral, notamos que nenhum dos valores de t é significante, mas que todos eles são relativamente grandes. Devido a esse fato é que obtivemos um erro entre, correspondendo ao conjunto, que era duvidoso. $\mathrm{Na}$ quadra $\AA$, dois valores de $t$ são bem significantes e a série formada pelas $\bar{v} p$ contem dois saltos : o limão cravo, com percentagem excessivamente baixa, de $36.48 \%$; e a laranja azeda, com percentagem relativamente alta, de $45,70 \%$, e separado dos demais cavalos; os outros 4 cavalos (limão rugoso, lima da Pérsia, laranja caipira e azeda-doce) praticamente são idênticos, variando a percentagem de polen vivo do cavaleiro apenas de 39,40 a 41,98 .

Uma comparação entre as médias parciais das duas quadras é impossivel, uma vez que a diferença entre elas mostrou ser significante, como dissemos atrás. Achamos importante referir que, como na experiência de Piracicaba, pudemos mais uma vez constatar diferenças significantes do polen do cavaleiro, provocadas pela ação do cavalo.

\section{IV - Análise do pomelo "Marsh-seedless":}

Esta experiência foi organizada e analisada como a da laranja pera (Mapa de plantação 3). Infelizmente, dada a seca reinante na ocasião, não floresceram as plantas enxertadas sobre o cavalo de tangerina cravo : 
mesmo assim, conseguimos determinar o polen de 164 enxertos, correspondentes a 32144 grãos de polen, na razão de 196 por lâmina.

Os resultados da decomposição do erro total para a percentagem do. polen vivo se encontram no quadro XI.

Antes de discutirmos com pormenores a decomposição, precisamos notar que o teste de homogeneidade, para este ensaio, contido no quadro XII (duas últimas colunas), não foi muito satisfatório. A variação dentro de um cavalo (laranja lima) era significantemente maior, e a variação de três cavalos (lima da Pérsia, laranja azeda-doce e trifoliata) significantemente menor que o erro experimental ou residual da experiência inteira. Entretanto, pareceu-nos acertado prosseguir a análise sem mais complicações, usando a-pesar-de tudo o erro residual. Ao diante, na análise individual dos cavalos, lembrar-nos-emos da heterogeneidade dos erros.

Voltando ao quadro XI, notamos que para o pomelo "Marsh-seedless" o terreno se mostrou bastante heterogêneo (quarta linha do quadro); especialmente na quadra $\AA$, a variação resultante desta fonte é quase da mesma dimensão que a do erro entre-cavalo; na quadra B, a-pesarde ser significante, é muito menor que o erro entre-cavalos. A variação entre-cavalos é bem grande nas duas quadras. A interação tambem é significante e da mesma ordem que o erro entre-cavalos na quadra $A$, e menor na quadra $B$.

A fusão das duas quadras deu o seguinte resultado: a diferença entre as quadras, medida pelo $\vartheta$, parece bem significante. Alem disso, há uma diferença profunda entre estas, que deixa dúvidas quanto à vantagem de se fundir, ou não, tal análise. Assim, das quatro componentes obtidas pela decomposição, apenas três (entre-repetições, interação cavalo-repetição e resíduo) são aproximadamente iguais ; a variaçãs entre-cavalos, por sua vez, difere muito entre si, pois, para a quadra $A$, o erro é de $\pm 9,62 \%$ e, para a quadra $B$, de $\pm 18,27 \%$.

$O$ estudo das médias parciais $\bar{v}$ p por cavalo (coluna 4, quadro XIII) demonstra que este erro entre-cavalo excessivamente alto, da quadra $B$, tem por causa principal a média do cavalo de Poncirus trifoliata, a qual é extremamente baixa e muito afastada da dos demais cavalos. Deixando fora o Poncirus julgamos que as médias parciais das duas quadras não parecem diferentes entre si, de um modo sistemático.

Eliminando completamente o cavalo de Poncirus trifoliata da análise, tanto da quadra $B$ como do conjunto $(A+B)$, podemos verificar (quadro XI, parte do meio) que a nossa conclusão era justificada. e:ro entre-cavalos cai, na quadra $B, a \pm 9,99 \%$ e fica assim praticamente 


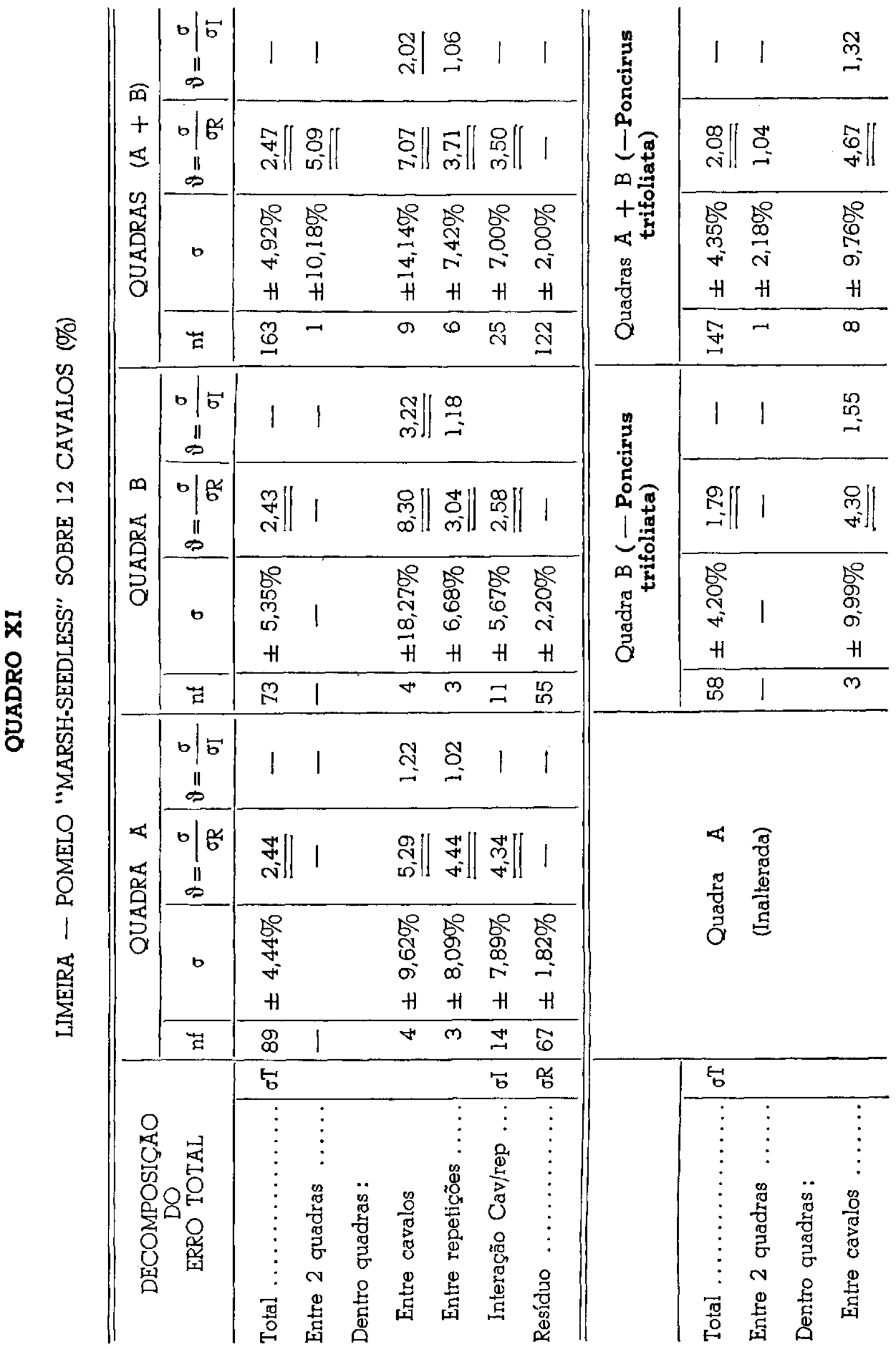




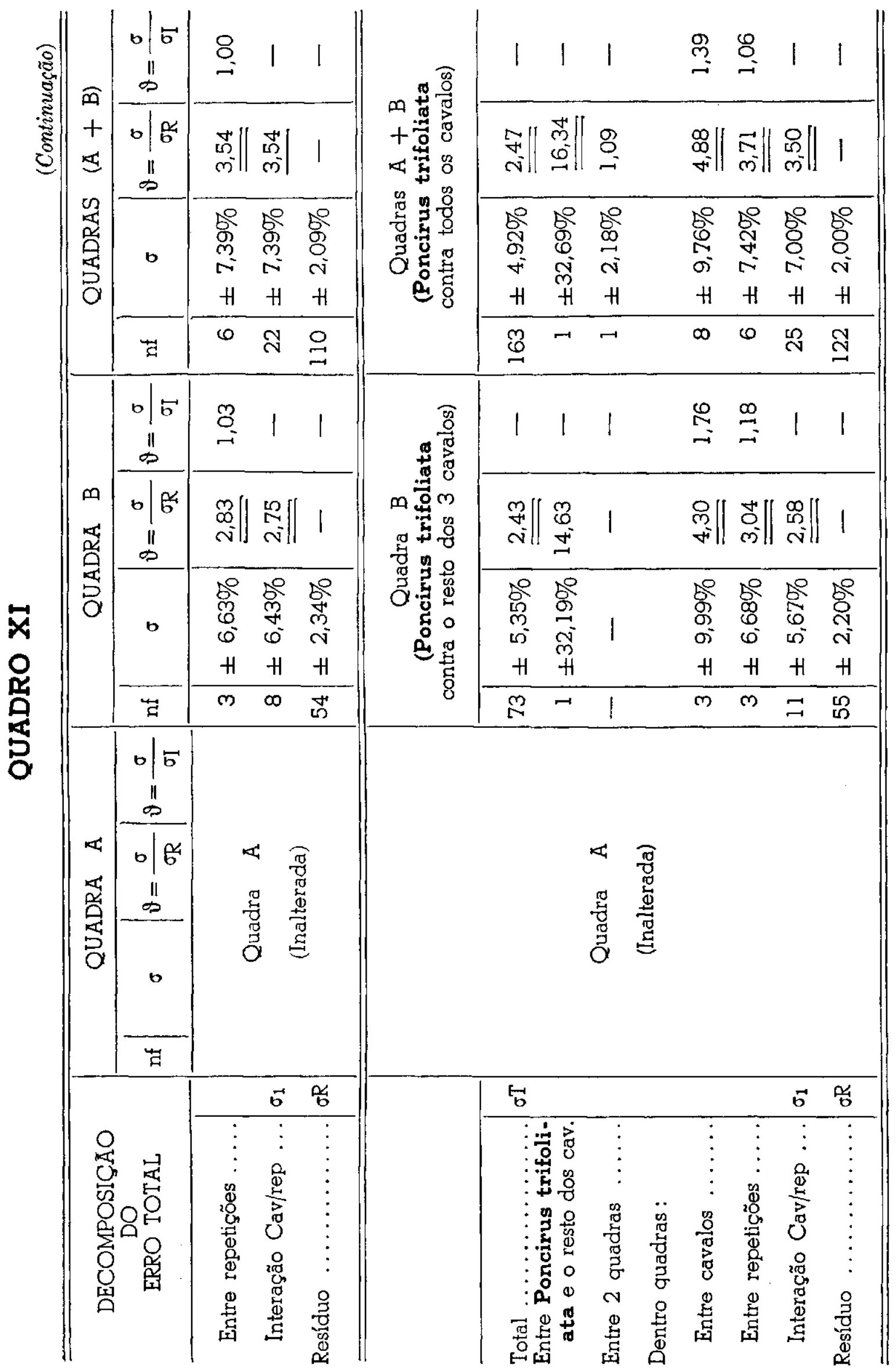




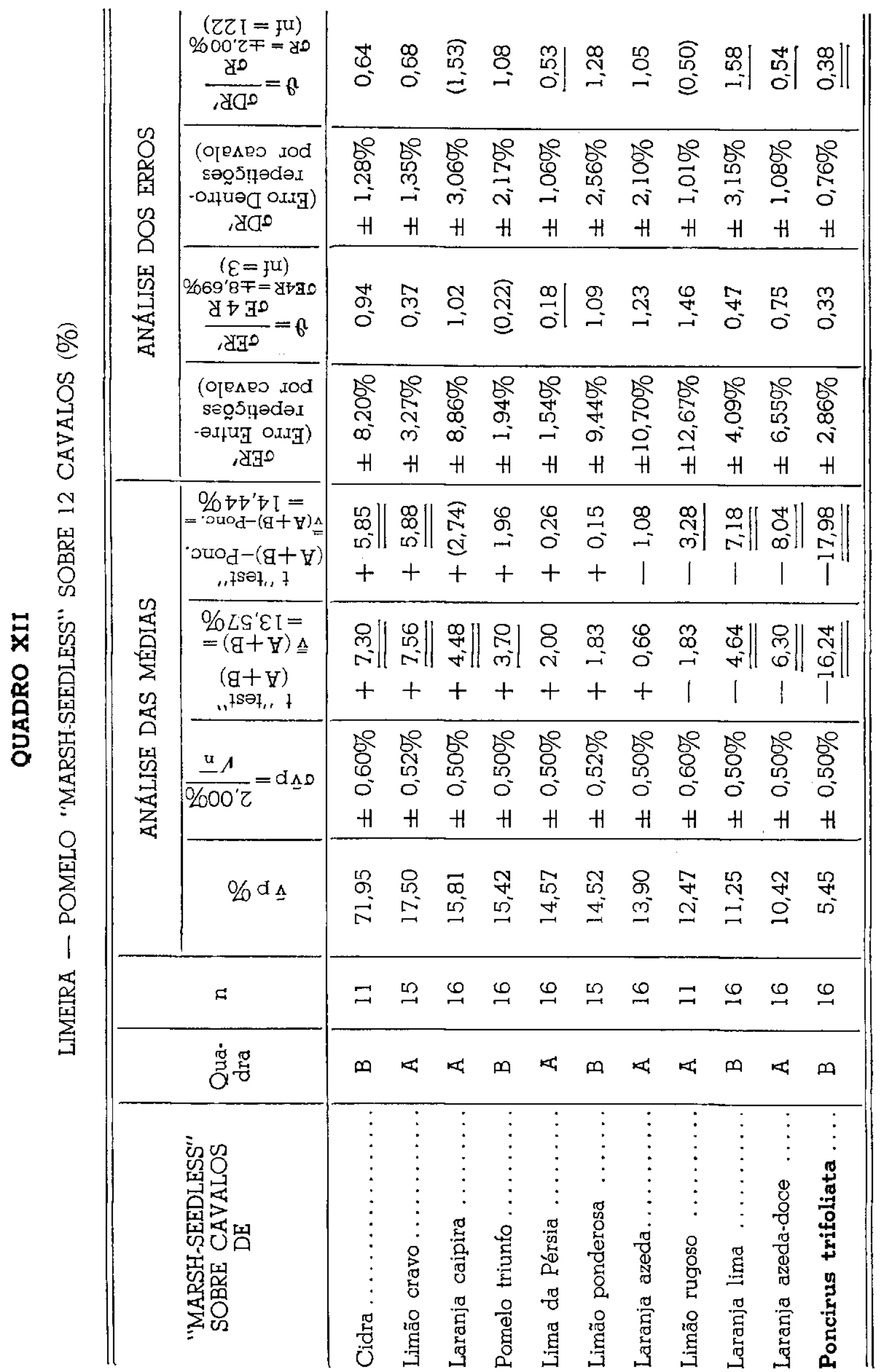


igual ao mesmo erro entre-cavalos da quadra $A$. $\bigcirc$ erro entre-quadras tambem cai muito, ficando insignificante $O s$ respectivos valores das médias do polen vivo por quadra (última coluna, quadro XIII) são, para $A$, de 14,18\%, e, para B (menos Poncirus), de 14,56\%.

\section{QUADRO XIII}

LIMEIRA - POMELO "MARSH-SEEDLESS" SOBRE 12 CAVALOS (\%)

\begin{tabular}{|c|c|c|c|c|c|c|}
\hline $\begin{array}{l}\text { Grupo } \\
\text { ou } \\
\text { Quadra }\end{array}$ & CAVALOS DE & $\begin{array}{l}\text { Repet. } \\
\quad 1 \\
\text { vp } \%\end{array}$ & $\begin{array}{c}\text { Repet. } \\
2 \\
\mathrm{vp} \%\end{array}$ & $\begin{array}{l}\text { Repet. } \\
3 \\
\text { vp } \%\end{array}$ & $\begin{array}{l}\text { Repet. } \\
\quad 4 \\
\text { vp } \%\end{array}$ & $\begin{array}{c}\overline{\mathrm{v}} \\
\text { horiz. } \\
\%\end{array}$ \\
\hline \multirow[t]{2}{*}{ A } & $\begin{array}{l}\text { Limão cravo } \ldots \ldots \ldots \ldots \ldots \\
\text { Laranja caipira } \ldots \ldots \ldots \ldots \\
\text { Lima da Pérsia } \ldots \ldots \ldots \ldots \\
\text { Laranja azeda } \ldots \ldots \ldots \ldots \\
\text { Limão rugoso } \ldots \ldots \ldots \ldots \\
\text { Laranja azeda-doce } \ldots \ldots \ldots\end{array}$ & $\begin{array}{r}16,64 \\
9,55 \\
13,48 \\
15,71 \\
19,27 \\
8,48\end{array}$ & $\begin{array}{r}18,21 \\
17,71 \\
15,24 \\
13,93 \\
6,70 \\
10,91\end{array}$ & $\begin{array}{l}19,44 \\
19,80 \\
14,62 \\
16,52 \\
11,44 \\
14,82\end{array}$ & $\begin{array}{r}15,72 \\
16,18 \\
14,94 \\
6,42 \\
\overline{7,46}\end{array}$ & $\begin{array}{l}17,50 \\
15,81 \\
14,57 \\
13,90 \\
12,47 \\
10,42\end{array}$ \\
\hline & $\overline{\mathrm{v}}$ vert. $\%$ & 14,36 & 13,78 & 16,11 & 14,12 & 14,18 \\
\hline \multirow[t]{2}{*}{ B } & $\begin{array}{l}\text { Cidra } \ldots \ldots \ldots \ldots \ldots \ldots \\
\text { Pomelo triunfo } \ldots \ldots \ldots \ldots \ldots \\
\text { Limão ponderosa } \ldots \ldots \ldots \ldots \\
\text { Laranja lima } \ldots \ldots \ldots \ldots \ldots \\
\text { Poncirus trifoliata } \ldots\end{array}$ & $\begin{array}{r}20,54 \\
16,28 \\
9,50 \\
13,36 \\
7,52\end{array}$ & $\begin{array}{r}20,18 \\
15,64 \\
18,56 \\
12,42 \\
5,02\end{array}$ & $\begin{array}{r}1 \overline{14,03} \\
18,73 \\
10,32 \\
5,02\end{array}$ & $\begin{array}{r}13,13 \\
15,74 \\
11,30 \\
8,90 \\
4,24\end{array}$ & $\begin{array}{r}17,95 \\
15,42 \\
14,52 \\
11,25 \\
5,45\end{array}$ \\
\hline & $\overline{\mathrm{v}}$ vert. $\%$ & 13,44 & 14,36 & 12,02 & 10,66 & 12,65 \\
\hline $\begin{array}{l}\text { B) - Ponc. } \\
\text { trifoliata }\end{array}$ & $\overline{\mathrm{v}}$ vert. $\%$ & 14,92 & 16,70 & 14,36 & 12,27 & 14,56 \\
\hline$A+B$ & $\overline{\mathrm{v}}$ vert. $\%$ & 13,94 & 14,05 & 14,47 & 11,40 & 13,57 \\
\hline$A+\underset{\text { trifoliata })}{B(- \text { Ponc. }}$ & $\overrightarrow{\mathrm{v}}$ vert. $\%$ & 14,58 & 15,45 & 15,52 & 12,20 & 14,44 \\
\hline
\end{tabular}

A eliminação de Poncirus, na quadra B, não tinha, porem, nenhum efeito sobre as outras três componentes dà decomposição: erros entrerepetições, interação cavalo-repetição e resíduo.

Tomando todos estes pontos em consideração, chegamos finalmente ao esquema da decomposição, que se acha na parte inferior do quadro XI, no qual, tanto para a quadra $B$ como para o conjunto $(A+B)$ a média parcial do Poncirus trifoliata é excluida do erro entre-cavalos; em compensação, aparecem um novo erro e $\vartheta_{t}$ correspondendo à diferença entre Poncirus e o grupo inteiro dos demais cavalos. Podemos, assim, entrar numa discussão minuciosa da decomposição contida no canto inferior direito do quadro XI. 
É notavel que o erro entre-repetições e o erro da interação cavalorepetição são bem significantes, e quase da mesma ordem que o erro entre-cavalos. A comparação dos erros entre-repetições por cavalo $\sigma E R^{\prime}$, que consta no quadro XII (8. ${ }^{\mathrm{a}}$ e 9. ${ }^{\mathrm{a}}$ colunas), foi feita esta vez em relação com o erro entre-repetições, e não com o erro residual, porquanto são diferentes entre si; podemos notar que há um valor de $\vartheta$ significante e pequeno demais (lima da Pérsia) e um duvidoso (pomelo triunfo). Parece dessa forma, que o terreno é igualmente heterogêneo para os demais cavalos.

Examinando-se com atenção a coluna dos erros entre-repetições por cavalo do quadro XII, verifica-se a existência de dois grupos : I) com erros bem pequenos, indo de $\pm 1,54$ a $\pm 4,09 \%$, composto dos cavalos de lima da Pérsia, pomelo triunfo, Poncirus trifoliala, limão cravo e laranja lima; II) com erros maiores, indo de $\pm 8,20$ a $\pm 12,67 \%$, formado pelos cavalos de cidra, laranja caipira, limão ponderosa, laranja azeda e limão rugoso. Há ainda o cavalo de laranja azeda-doce, cujo erro, por estar bem no meio da série, $\pm 6,55 \%$, dificulta sua inclusão em qualquer dos dois grupos.

Para vermos como estes dois grupos se comportam, podemos calculàr - erro balançado entre-repetições por cavalo, para ambos, e depois compará-los com o erro residual. O grupo I dá um erro balançado de $\pm 2,89 \%$ e o grupo II de $\pm 10,05 \%$. Fazendo a comparação com o erro residual da experiência de $\pm 2,00 \%$, dá, para o grupo I, um valor de $\vartheta$ duvidoso e, para o grupo II, um $\vartheta$ muito significante. Disto concluimos que há uma variação exagerada neste último grupo de cavalos, a qual podemos atribuir à diferença de época na colheita das flores, ou ainda a manchas especiais do terreno, que afetam as repetições de modos diversos, produzindo interação.

$O$ efeito da interação pode ainda ser notado no quadro XIII, que contem as médias parciais $\bar{v} p$ por cavalo e repetição; por esse quadro, percebemos que os diferentes cavalos apresentam bastantes divergências. Por exemplo, na quadra $\mathrm{B}$, ○ limão ponderosà tem o valor máximo na repetição 1 e o mínimo na repetição 4 . Na quadra $A$, a laranja caipira tem o valor máximo na repetição 3 e o mínimo na repetição 1 ; a laranja azẹda tem o máximo na repetição 1 e o mínimo na repetição 4.

A vista do exposto, não podemos dar explicação segura, fisiológica ou mesológica, destas divergências. Contudo, devemos lembrar que já notamos efeitos semelhantes para os mesmos cavalos, quando enxertados com laranja pera, sendo que, para o pomelo "Marsh-seedless", a heterogeneidade do terreno foi ainda mais acentuada.

A análise das médias parciais por cavalo pode ser feita para as duas 
quadras, em conjunto, uma vez que a decomposição não mostrou diferenças entre elas, após a eliminação do cavalo de Poncirus trifoliata. Os valores das $\bar{v}$ p por cavalo e os de $t$ são dados, respectivamente, na 4." e 7.a colunas do quadro XII. Podemos ver que as $\overrightarrow{\mathrm{v} p}$ dos cavalos formam série contínua, indo de um máximo de polen vivo do cavaleiro de $17,95 \%$ para o cavalo de cidra, até um mínimo de $10,42 \%$ para o cavalo de laranja azeda-doce. Nesta série de 10 médias, duas de cada extremo teem valores de $\mathbf{t}$ fora de $1 \%$ limite de probabilidade, 0 que naturalmente não pode ser efeito do acaso.

$O$ fato de o cavalo de Poncirus trifoliata dar um valor $\mathbf{t}$ de ordem completamente diferente dos demais cavalos exclue a possibilidade de que pertença a esta mesma série, o que, ademais, já comprovamos anteriormente.

Querendo dar mais uma prova de que o Poncirus não pertence à série dos 10 cavalos, fizemos um teste da sua média para a menor média da série, que é a do cavalo de laranja azeda-doce. Esse teste dá um valor muito significante, bem fora do $1 \%$ limite de probabilidade:

$$
\mathbf{t}=\frac{\overline{\mathrm{v}}_{1}-\overline{\mathrm{v}}_{2}}{\sigma \overline{\mathrm{v}} \text { dif }}=\frac{\overline{\mathrm{v}}_{1}-\overline{\mathrm{v}}_{2}}{\sqrt{\frac{\sigma_{1}{ }^{2}}{\mathrm{n}_{1}}+\frac{\sigma_{2}{ }^{2}}{\mathrm{n}_{2}}}}=\frac{10,45-5,45}{\sqrt{\frac{(1,08)^{2}}{16}+\frac{(0,76)^{2}}{16}}}=+15,06 \frac{\mathrm{nf}_{1}=1}{\mathrm{nf}_{2}=30}
$$

Se analisarmos cuidadosamente a série de médias parciais dos cavalos para o pomelo "Marsh-seedless", como consta no quadro XII, iremos notar que elas se aproximam, comparativamente, do limite zero ; pareceunos possivel que daí resultassem certas anomalias da distribuição, isto é, certa assimetria.

A-fim-de evitar a pressão do limite zero em tais casos, há uma transformação das percentagens em ângulo, proposta por Fisher, segundo Clark e Leonardo (3). Essa transformação consiste em substituir os valores de a\% pelo seguinte valor de $\mathrm{x}$ :

$$
x= \pm \sqrt{\text { seno }\left(\frac{a \%}{100}\right)}
$$

onde o valor de $x$, a-pesar-de ser ângulo, e dever, portanto, ser expresso na dimensão de graus, é escrito como número sem denominação. Alem disso, as frações de graus (sistema sexagesimal) são expressas em decimais (sistema decimal).

Sobre este importante ponto da transformação das percentagens em ângulo, temos em preparo outra publicação (Brieger e Gurgel).

Com os valores das percentagens transformadas em ângulo, repetimos novamente o processo inteiro da decomposição, dado nos quadros $\mathrm{XIV}, \mathrm{XV}$ e XVI. O resultado, porem, altera as conclusões de um modo 


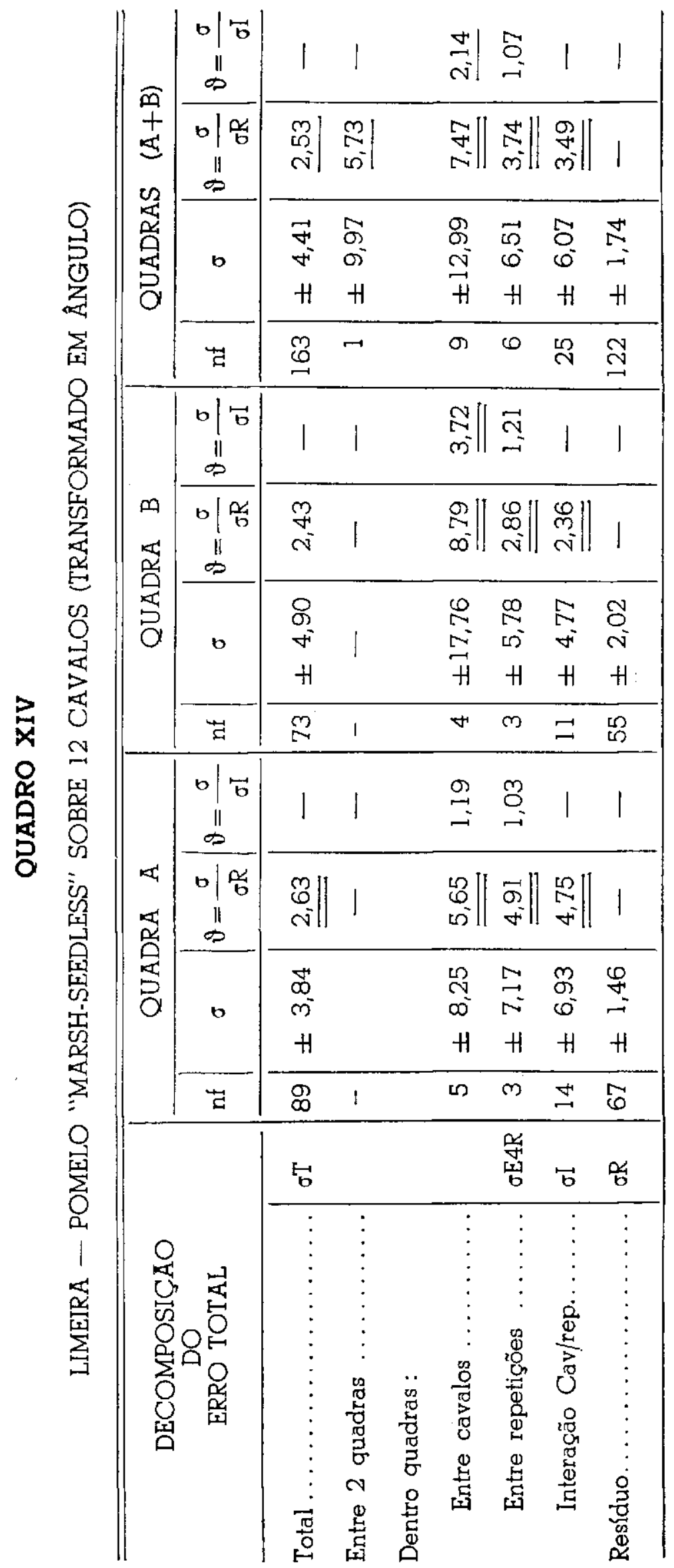




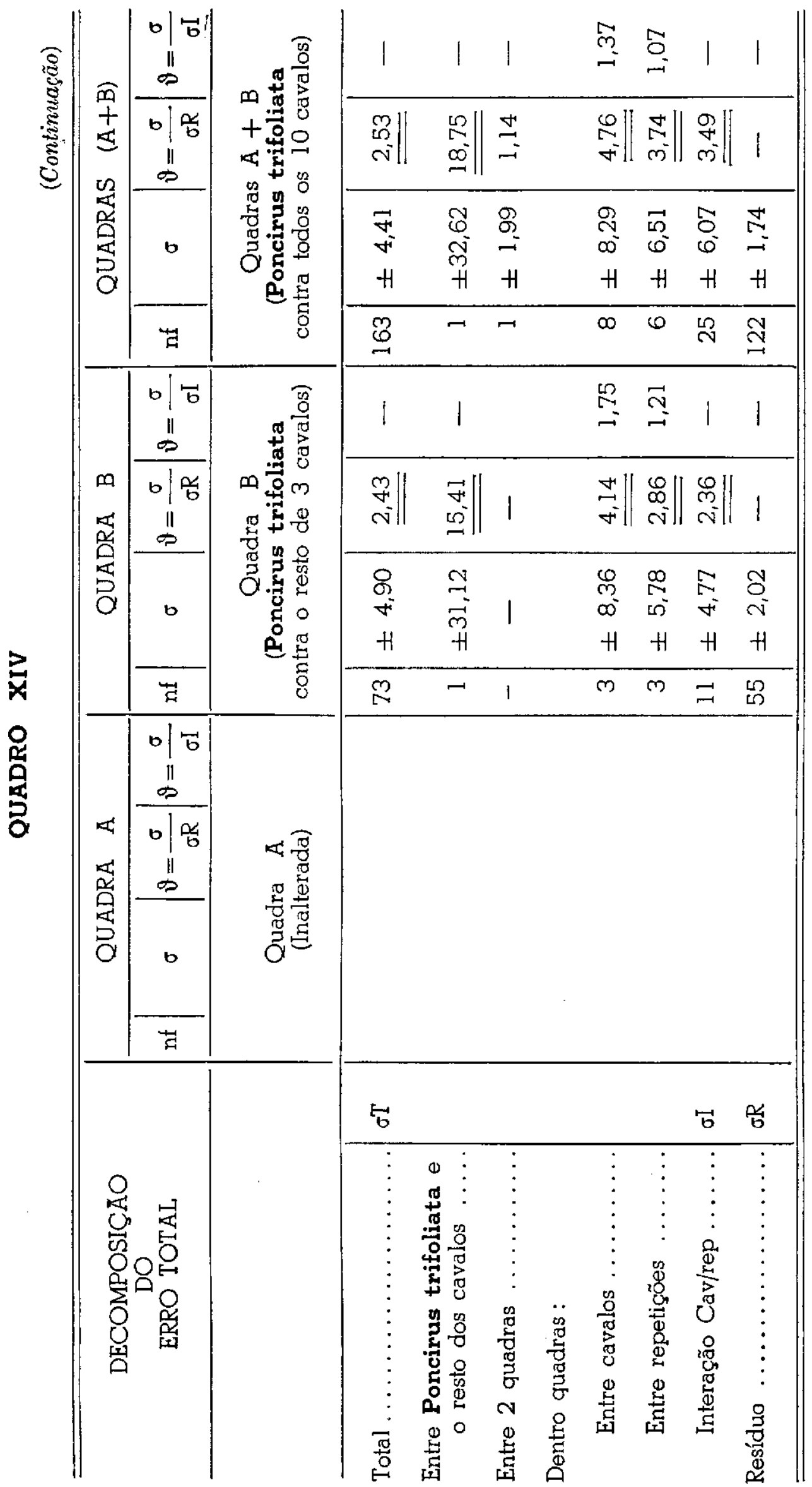




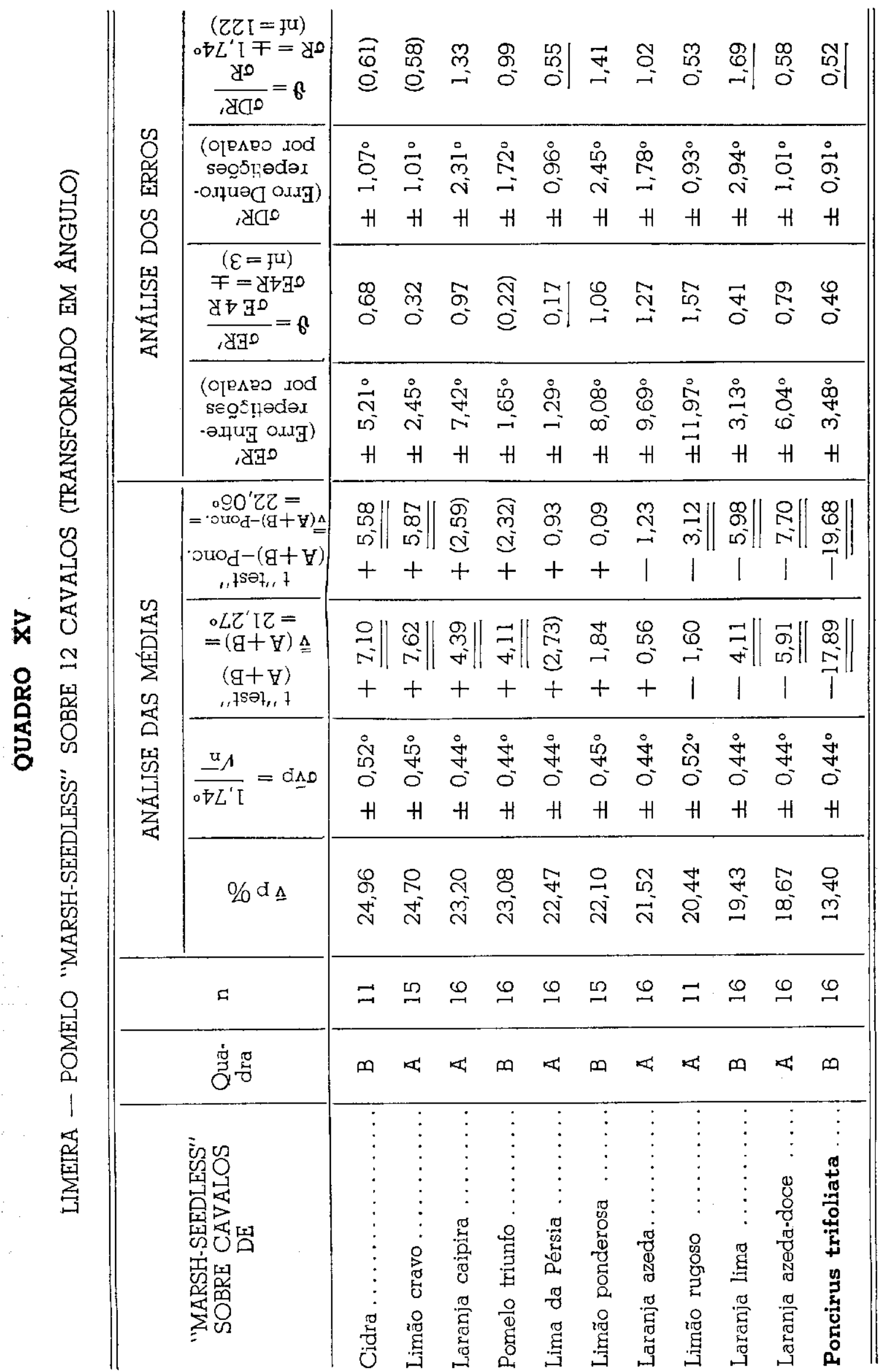


bastante limitado. A diferença entre Poncirus trifoliata e os demais cavalos fica ainda mais acentuada. Ot teste das médias parciais por cavalo, dado na coluna 7 do quadro XV, sofreu pequena modificação; assim, a média do cavalo de limão rugoso, que deu, sem transformação, um $\mathbf{t}$ entre $1 \%$ e $1 \%$ limite de probabilidade, passa agora a ser fora do $1 \%$ limite, e o valor para o cavalo de pomelo triunfo, que tinha um $\mathbf{t}$ teste significante, passa a ter valor apenas entre $5 \%$ e $1 \%$ limite de probabilidade.

Acentuou-se tambem um pouco a heterogeneidade geral da experiência, medida pelo erro residual por cavalo, como se pode ver pela comparação das últimas colunas dos quadros XV e XVI.

\section{QUADRO XVI}

LIMEIRA - POMELO "MARSH-SEEDLESS" SOBRE 12 CAVALOS (TRANSFORMADO EM ÂNGULO).

\begin{tabular}{|c|c|c|c|c|c|c|}
\hline $\begin{array}{l}\text { Grupo } \\
\text { ou } \\
\text { Quadra }\end{array}$ & $\begin{array}{c}\text { "MARSH-SEEDLESS" } \\
\text { SOBRE CAVALOS } \\
\text { DE }\end{array}$ & $\begin{array}{l}\text { Repet. } \\
1 \\
\overline{\mathrm{v}} \mathrm{p}^{\circ}\end{array}$ & $\begin{array}{l}\text { Repet. } \\
2 \\
\overline{\text { vpo }}\end{array}$ & $\begin{array}{c}\text { Repet. } \\
3 \\
\overrightarrow{v p}^{\circ}\end{array}$ & $\begin{array}{l}\text { Repet. } \\
\quad 4 \\
\bar{v} p^{\circ}\end{array}$ & $\begin{array}{c}\overline{\mathrm{v}} \\
\text { horizo }\end{array}$ \\
\hline \multirow[t]{2}{*}{ A } & $\begin{array}{l}\text { Limão cravo } \ldots \ldots \ldots \ldots \\
\text { Laranja caipira } \ldots \ldots \ldots \ldots \\
\text { Lima da Pérsia } \ldots \ldots \ldots \ldots \\
\text { Laranja azeda } \ldots \ldots \ldots \ldots \\
\text { Limão rugoso } \ldots \ldots \ldots \ldots \\
\text { Laranja azeda-doce } \ldots \ldots \ldots\end{array}$ & $\begin{array}{l}24,07 \\
17,95 \\
21,52 \\
25,60 \\
26,60 \\
16,93\end{array}$ & $\begin{array}{l}25,25 \\
24,82 \\
22,96 \\
21,91 \\
14,95 \\
19,30\end{array}$ & $\begin{array}{l}26,13 \\
26,33 \\
22,65 \\
23,95 \\
19,78 \\
22,64\end{array}$ & $\begin{array}{l}23,33 \\
23,69 \\
22,76 \\
14,61 \\
-15,81\end{array}$ & $\begin{array}{l}24,70 \\
23,20 \\
22,47 \\
21,52 \\
20,44 \\
18,67\end{array}$ \\
\hline & $\overline{\mathrm{v}}$ vert. $^{\circ}$ & 22,11 & 21,53 & 23,58 & 20,04 & 21,83 \\
\hline \multirow[t]{2}{*}{ B } & $\begin{array}{l}\text { Cidra } \ldots \ldots \ldots \ldots \\
\text { Pomelo triunfo } \ldots \ldots \ldots \ldots \\
\text { Limão ponderosa } \ldots \ldots \ldots \\
\text { Laranja lima } \ldots \ldots \ldots \\
\text { Poncirus trifoliata } \ldots \ldots\end{array}$ & $\begin{array}{l}26,97 \\
23,80 \\
17,61 \\
21,34 \\
15,90\end{array}$ & $\begin{array}{l}26,71 \\
23,29 \\
25,52 \\
20,48 \\
12,94\end{array}$ & $\begin{array}{l}-\overline{21,90} \\
25,63 \\
18,57 \\
12,92\end{array}$ & $\begin{array}{l}21,20 \\
23,34 \\
19,62 \\
17,34 \\
11,86\end{array}$ & $\begin{array}{l}24,96 \\
23,08 \\
22,10 \\
19,43 \\
13,40\end{array}$ \\
\hline & $\overline{\mathrm{v}}$ vert. ${ }^{\circ}$ & 21,12 & 21,79 & 19,76 & 18,67 & 20,59 \\
\hline B (- Ponc. & $\overline{\mathrm{v}}$ vert. ${ }^{\circ}$ & 21,43 & 24,00 & 22,03 & 20,38 & 22,39 \\
\hline$A+B$ & $\overline{\mathrm{v}}$ vert.o & 21,66 & 21,65 & 22,05 & $19,36^{\circ}$ & 21,27 \\
\hline $\begin{array}{c}A+B(-P o n- \\
\text { cirus) } \ldots \ldots\end{array}$ & $\overrightarrow{\mathrm{v}}$ vert.o & 22,34 & 22,52 & 23,06 & 20,19 & 22,06 \\
\hline
\end{tabular}

De um modo geral, o efeito da transformação das percentagens em ângulo, em nossa experiência de cavalos para o pomelo "Marsh-seedless", não teve muita importância. 


\section{V - Análise da laranja Baianinha Piracicaba :}

Mais uma vez, como já aconteceu na experiência de Piracicaba, a análise da laranja Baianinha Piracicaba sobre os 12 diferentes cavalos mostrou uma esterilidade completa do polen, em todas as combinações.

\section{G) - DISCUSSÃO}

\section{I - Homogeneidade do método :}

No teste contido no capítulo $\mathrm{D}$ deste trabalho, verificamos, de modo geral, que o método de colher o polen, preparar as lâminas e executar as contagens foi satisfatório.

Para este fim, fizemos uma comparação, pelo $\vartheta$ teste, entre o erro determinado diretamente das contagens e o erro ideal oi, calculado pela fórmula teórica, na hipótese de que as determinações do polen fertil devem mostrar distribuição normal e binomial, em volta de sua média.

Para o cálculo do erro ideal, usamos a fórmula:

$$
\sigma i= \pm \sqrt{\frac{\overline{\hat{\mathrm{v}} \%(100-\overline{\mathrm{v}} \%)}}{\mathrm{n}}}
$$

onde $\overline{\mathrm{v}}$ é a média das determinações ou contagens e $\mathrm{n}$ o número médio de grãos de polen contados por lâmina. Com o uso deste último valor, introduzimos certa inexatidão, uma vez que o número de grãos de polen contados por lâmina não foi sempre o mesmo, mas variou entre os extremos de 72 a 343. Como o erro observado, usamos o erro residual $\sigma R$, determinado pela fórmula:

$$
\sigma R= \pm \sqrt{\frac{\Sigma_{\mathrm{v}^{2}}-\Sigma_{\mathrm{C}^{1}}}{\mathrm{n}-\mathrm{m}}}
$$

Podíamos neste caso ter usado o erro total oT, uma vez que a decomposição não deu nenhum sinal de heterogeneidade. $O$ erro total é, por sua vez, definido pela fórmula:

$$
\sigma \mathrm{T}= \pm \sqrt{\frac{\Sigma \mathrm{v}^{2}-\mathrm{c}}{\mathrm{nf}}}
$$

Obtemos assim os dois valores seguintes de $\vartheta$ :

$$
\begin{array}{ll}
\vartheta=\frac{\sigma \mathrm{R}}{\sigma \mathrm{i}}=\frac{4,07}{3,23}=1,26 \text { (insignificante) } & \frac{\mathrm{nf}_{1}=30}{\mathrm{nf}_{2}=\infty} \\
\vartheta=\frac{\sigma \mathrm{T}}{\sigma \mathrm{i}}=\frac{3,94}{3,23}=1,22 \text { (insignificante) } & \frac{\mathrm{nf}_{1}=44}{\mathrm{nf}_{2}=\infty}
\end{array}
$$


Podemos agora fazer tambem a mesma comparação para as demais experiências. Mas, já que em todos esses casos a decomposição do erro total demonstrou a existência de fortes heterogeneidades, a comparação pelo $\vartheta$ teste só pode ser feita usando os erros residuais. $O$ número médio de grãos de polen por lâmina e por ensaio, a ser usado na fórmula do erro ideal, e tambem os extremos, serão os seguintes:

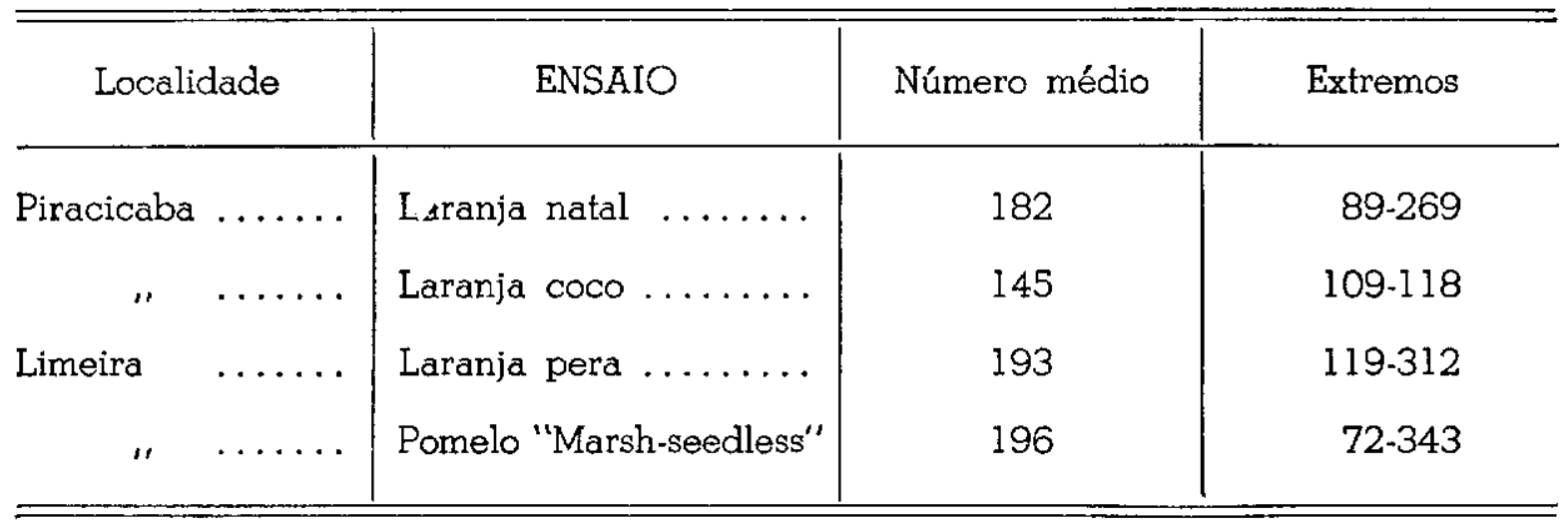

Obtivemos assim os 4 valores seguintes de $\vartheta$ :

\begin{tabular}{|c|c|c|c|c|}
\hline Localidade & ENSAIO & $\vartheta=\frac{\sigma \mathrm{R}}{\sigma \mathrm{i}}$ & Significância & $\begin{array}{c}\text { Graus } \\
\text { de } \\
\text { liberdade }\end{array}$ \\
\hline \multirow{4}{*}{ Piracicaba } & Laranja natal & $\vartheta=\underline{5,66}=$ & Fora de $1 \%$ & $\mathrm{nf}_{1}=39$ \\
\hline & & 3,65 & & $\mathrm{nf}_{2}=\infty$ \\
\hline & Laranja coco & $\vartheta=\underline{7,15}=1,89$ & Idem & $\mathrm{nf}_{1}=40$ \\
\hline & & 3,78 & & $\mathrm{nf}_{2}=\infty$ \\
\hline \multirow[t]{3}{*}{ Limeira } & Laranja pera & $\vartheta=\frac{4,51}{250}=1,27$ & Idem & $\mathrm{nf}_{1}=118$ \\
\hline & Pomelo "Marsh. & $\begin{array}{l}3,50 \\
2,00\end{array}$ & & $\begin{array}{l}n f_{2}=\infty \\
n f_{1}=122\end{array}$ \\
\hline & seedless" & $\vartheta=\frac{2,00}{2,45}=0,82$ & Entre $1 \%$ e $1 \%$ & $\frac{\mathrm{nI}_{1}=124}{\mathrm{nf}_{2}=\infty}$ \\
\hline
\end{tabular}

É evidente que todos os quocientes $\vartheta$ são significantes, o que parece, à primeira vista, indicar variações bastante fortes nos três primeiros casos e muito uniformes no últímo. Mas, não devemos esquecer que o $\vartheta$ teste com graus de liberdade elevados é muito sensivel, de maneira que a segunda casa decimal já é de ordem decisiva, e que o número de grãos de polen por lâmina era extremamente variavel, notadamente nas amostras de Limeira. Isto nos leva a crer que o valor médio usado (dos grãos de polen) representa uma aproximação bastante imperfeita, o que nos deixa em dúvida sobre se as primeiras casas decimais foram suficientemente calculadas. 
Devido a este último fato, não devemos atribuir muito peso aos quocientes $\vartheta$ da experiência de Limeira, onde os erros residuais quase não diferem do erro ideal. Todavia, já em Piracicaba, os erros residuais diferem em várias unidades do erro ideal, sendo que, neste caso, os valores significantes de $\vartheta$ não podem mais ser atribuidos à inexatidão do cálculo.

Levando tudo isto em consideração, chegamos a concluir que as variações do erro ideal em Limeira podem ser unicamente atribuidas ao acaso, enquanto que as de Piracicaba são, com certeza, demasiadamente grandes.

De-fato, não ficaríamos admirados se encontrássemos uma variação maior do que a esperada unicamente pelo acaso, uma vez que não nos foi possivel fazer a colheita das flores e preparação das lâminas em condições muito uniformes. Como explicamos no capítulo $C$, os galhinhos com botões eram colhidos no pomar, transportados a laboratório e, somente depois de abrirem as flores e as anteras, é que preparávamos as lâminas, fazendo então a contagem. Ainda mais : a colheita do material da Estação Experimental de Limeira não foi feita de uma só vez, mas estendeu-se aproximadamente durante dois meses, pelo fato de a floração não ter sido uniforme.

As condições de colheita em Piracicaba foram mais uniformes, contribuindo para isso a maior uniformidade da floração, pois, necessitamos de apenas uma semana para colher todo o material ; sobretudo, em vista da proximidade entre o pomar e o laboratório, o dificultoso transporte ficou abolido. Estamos então inclinados a atribuir a variação exagerada, encontrada em Piracicaba, mais a uma heterogeneidade inerente às plantas do que ao método de colheita.

Em consequência da falta de repetições, não nos foi possivel provar esta última hipótese.

\section{II - Efeito do terreno :}

Seria interessante resumir agora o que ficou comprovado sobre o efeito do terreno, com relação aos ensaios de Limeira (quadros VIII e XI).

Para o ensaio da laranja pera (última parte do quadro VIII), vimos que não havia um erro significante entre repetições e demonstramos que as quadras foram razoavelmente uniformes entre si. A diferença significante entre as mesmas demonstra, porem, que a fertilidade do polen é afetada pelas variações do terreno. Concluimos, ademais, que as quadras não apresentavam manchas menores que a própria área. 
Para o pomelo "Marsh-seedless" (canto inferior direito do quadro $\mathrm{XI})$, a existência de um erro significante entre repetições indica claramente que o terreno era manchado. Da ausência de uma diferença significante entre-quadras, podemos concluir que as manchas foram igualmente distribuidas em todo o terreno, contrabalançando-se assim seu efeito dentro da quadra.

É de muita importância e de interesse geral que, para um carater bem especializado como a fertilidade do polen, possamos notar um efeito estatisticamente significante de desigualdade do terreno. Pareceu-nos justificado generalizar esta conclusão e dizer que, para outros fatores fisiológicos do cavaleiro, devemos esperar tambem efeitos correspondentes. Daí deduzirmos que é indispensavel executar experiências de modo que permitam sempre análise de homogeneidade, ou seja, em linhas gerais, uma execução de experiências com repetições.

\section{III - Efeito do cavalo sobre o cavaleiro :}

Ficou provado, em todas as experiências anteriormente discutidas, que há uma influência do cavalo sobre o cavaleiro, no concernente à fertilidade do polen. Desde que o mesmo cavalo foi usado com vários cavaleiros, poderemos analisar se tem sempre, ou pelo menos preferivelmente, um idêntico efeito.

Nos quadros V, VII, X e XIII encontramos, nas últimas colunas, as percentagens médias, organizadas em ordem decreccente. Notamos assim que o cavalo de laranja azeda, usado nos quatro ensaios, tem, num caso, quando combinado com o cavaleiro de pera (quadro IX), efeito significante, aumentando a fertilidade do polen. Nos outros ensaios o efeito foi insignificante do ponto de vista da estatística. Com o pomelo "Marsh-seedless" e laranja coco, porem, ele causou redução de fertilidade do polen.

O cavalo de lima da Pérsia em nenhum caso deu efeito significante, sendo que, para os cavaleiros do pomelo "Marsh-seedless" e laranja pera, a percentagem média do polen ficou perto da média da série. Para a laranja coco a percentagem do polen vivo foi a primeira da série, e, para a laranja natal, a última.

O cavalo de limão rugoso dá, em um caso apenas, efeito significante em combinação com o pomelo "Marsh-seedless", reduzindo a fertilidade do polen. Nos outros três ensaios achamos, mais uma vez, irregularidades, sendo a fertilidade bem grande para laranja coco e natal, e média, para a pera. 
É interessante mencionar aquí o caso do cavalo de Poncirus trifoliata, a-pesar-de ter sido usado apenas na experiência de Limeira. Com o enxerto do pomelo "Marsh-seedless", ele causou redução muito significante da fertilidade do polen, enquanto que para a laranja pera ficou sem nenhum efeito. Esta dupla ação é especialmente notavel, uma vez que se trata de cavalo oriundo de material provavelmente uniforme e de mesma fonte de origem.

Nesses quatro casos analisados não podemos encontrar nenhuma coerência, mas não devemos esquecer de que se trata, com exceção, de médias que não diferem significantemente das respectivas médias gerais.

Podemos ainda usar, para essa comparação, dados de outra experiência, (19) sobre a fertilidade do polen em várias espécies è variedades do gênero Citrus. Nesta experiência foram incluidas determinações do polen vivo de vários cavaleiros sobre os cavalos de laranja azeda e limão rugoso, de conformidade com o seguinte quadro:

\begin{tabular}{|c|c|c|c|}
\hline \multirow{2}{*}{ Localidade } & \multirow{2}{*}{ Cavaleiro } & \multicolumn{2}{|c|}{ Cavalo de } \\
\hline & & $\begin{array}{c}\text { Laranja } \\
\text { azeda }\end{array}$ & $\begin{array}{l}\text { Limão } \\
\text { rugoso }\end{array}$ \\
\hline $\begin{array}{c}\text { Piracicaba } \\
\text { ", } \\
\text { ", } \\
\text { ", } \\
\text { ", }\end{array}$ & $\begin{array}{ll}\text { Laranja } & \text { Hamlim } \\
\text { " } & \text { Pineapple } \\
\text { " } \quad \text { Ruby } \\
\text { L' Valencia Late } \\
\text { Limão galego } \\
\text { Temple-orange }\end{array}$ & $\begin{array}{l}69,6 \% \\
79,3 \% \\
66,3 \% \\
31,5 \% \\
58,4 \% \\
61,7 \%\end{array}$ & $\begin{array}{l}77,1 \% \\
75,0 \% \\
74,9 \% \\
37,6 \% \\
43,6 \% \\
61,1 \%\end{array}$ \\
\hline
\end{tabular}

Observamos aquí a mesma irregularidade já anteriormente mostrada. Em três casos, a percentagem do polen vivo sobre limão rugoso é maior do que sobre laranja azeda, e, em dois outros casos, é justamente o contrário; no último exemplo, a percentagem é quase igual para os dois cavalos.

De todas estas observações notamos que os efeitos do cavalo sobre a fertilidade do polen do cavaleiro não são dirigidos de modo sistemático. Com certeza não se trata aquí de ação unilateral e simples do cavalo, mas a fertilidade do polen deve ser evidentemente determinada por uma ação conjunta entre certos cavalos em combinações com certos cavaleiros. 
Temos, porem, de restringir um pouco a generalidade desta conclusão. Os cavalos usados aquí em nosso país, em hipótese alguma, representam material homogêneo. São sempre obtidos de mistura de sementes de diversas origens e ainda não controladas. Torna-se, portanto, impossivel estabelecer quais as plantas que deram origem aos cavalos utilizados nos ensaios. Alem disso, deveria ser tomada em consideração qual a frequência de produzir embriões sexuais ou apogâmicos, nas referidas espécies de cavalos. Seria interessante repetir as mesmas experiências mais tarde, quando já se tenham estabelecido linhas puras ou clones de cavalos, pois, pode ser que diferentes linhagens de uma mesma espécie de cavalo se comporte de modo diverso quanto aos diferentes cavaleiros, umas dando efeitos e outras não.

De passagem, seria bom lembrarmos que nas experiências de Piracicaba e Limeira não havia nenhuma influência do cavaleiro, pois formavam clones, em virtude de terem sido retirados sempre de uma mesma planta. É ainda interessante notar que, a-pesar da semelhança que há entre a laranja natal, de Piracicaba, e pera, de Limeira, elas mostraram ser completamente diferentes no comportamento, com relação à fertilidade do polen.

\section{H) - CONCLUSÕES}

1 - A técnica usada (colheita, preparação e contagem das lâminas) parece bastante satisfatória, de acordo com os resultados da análise estatística.

2 - A fertilidade do polen do cavaleiro, em enxertia, é, em parte, dependente do terreno onde as plantas se acham, e necessita, portanto, de uma análise estatística pormenorizada, de ensaios com repetições.

3 - Os cavalos podem ter efeito sobre a fertilidade do polen do cavaleiro, seja produzindo aumento ou redução, estatisticamente significantes em alguns casos, e sem nenhum efeito noutros. Esse efeito, porem, não parece sistemático ; uma espécie de cavalo em combinação com diferentes cavaleiros pode dar diferentes resultados.

\section{I) - AGRADECIMENTOS}

É justo que deixemos aquí registados agradecimentos sinceros aos nossos colegas G. O. N. Addison e Nahum Klein, pela valiosa ajuda nas contagens do polen, e ao sr. Eduardo Rodrigues A., pelo auxílio nos cálculos estatísticos. 


\section{SUMMARY}

1 - The object of the present paper is the study of the effect of different rootstocks on pollen fertility of the scion. The material was obtained from experiments by Moreira (18), in progress at the Experimental Station, Limeira, São Paulo, and from experiments by Vasconcelos (1), in progress in the Experimental Orchard at Piracicaba, São Paulo.

\section{Homogeneity test of method}

2 - A preliminary homogeneity test was carried out and showed that the methods for collecting and counting pollen were sufficiently satisfactory.

This test consisted in the following: pollen was collected from 3 plants for each of 5 different rootstocks, budded with laranja pera and for each plant 3 determinations were made. The decomposition of the total error showed no significant, large component (quadro I). Both error and means per plant or rootstock gave no signs of heterogeneity (quadros II and III). Finally the error of a binomial distribution around a mean of $40,19 \%$ and with an average of 145 pollen grains per determination, amounted to $\pm 3,23 \%$, value pratically identical with that actually obtained ( $\pm 4,07$ in quadro I).

\section{Experiments in Piracicaba}

3 - The Experiment at Piracicaba included 3 commercial varieties of oranges: "Baia", "natal" and "coco", budded on five rootstocks (see quadros V and VII). The lay out of the experiment did not include any systhematic or random repetitions; 10 plants of each combination were planted in one row each, as indicated in graph 1 .

4 - The plants of "Baia" could not be included in the analysis since they did not contain any pollen. The determination of pollen fertility from the other two scions were subjected to an analysis of variance. In order to compensate for the lack of replications in the original lay out, 3 pollen counts were made for each individual plant. Thus the tota error could be divided into three parts: between rootstocks, between plants-within rootstocks, between determinations within plants (quadros IV and VI). Since in each of the two cases the last two components did not differ, they were united into one residual error "within rootstock".

No differences between pollen fertility caused by different rootstock, could be detected for "natal" (quadros IV and V), while in the ca se of 
"coco" (quadros VI and VII) there is some indication for sour orange (laranja azeda) of a slight reduction from ca $70 \%$ of normal grains to only $64 \%$, with error standard for the latter mean of $\pm 2,38 \%$.

\section{Layout of experiment in Limeira}

5 - The layout of the experiment at Limeira was much more complete and included four complete randon replications. Since part of the plots contained 4 individuals and others 9 , the plots with 4 and 9 trees were planted separatly and their results were afterwards combined by confounding. The complete layout has been described by Moreira (18).

The experiment included again three commercial varieties namely : the grape-fruit "marsh seedless" and the sweet oranges "pera" and "Baía". Of these, "Baía" was completely male sterile, while the other two produced viable pollen.

6 - The analysis for each sub-bloc (or "quadra") consisted in a decomposition of the total error into four parts: between rootstocks, between repetitions, interaction rootstock-repetition and finally the residual error within plots. After confounding, one more component appears: between sub-blocs or quadras (quadros VIII and XI).

7 - The residual error was subjected to an homogeneity test. The individual errors "within repetition per rootstock" and their respective values $\vartheta$ are given in the last columns of quadros IX and XII. In the first case all are insignificant, while in the latter there is a certain heterogeneity. There are three significant values of $\vartheta$, one larger and two smaller than 1, beside the very extreme value obtained for Poncirus trifoliata which, however, has to be treated separatly as we shall see later.

\section{Orange "pera" Limeira}

8 - The decomposition of the total error in each "quadra" or subbloc for "pera" gave the following results: There is a significantly high variation between rootstocks in sub-bloc $A$ and a probably excessive variation between rootstocks in sub-bloc $B$. It seems that there is no difference between repetitions, except for a significant interaction in sub-bloc B. Since pratically all the components and especially the residual errors of the two sub-blocs are of the same order, the two subblocs were united and as result a new component appears: "between sub-blocs", which is highly significant. The significance of the errors 
"between rootstock" and also of the interaction remain unaltered (quadro VIII).

9 - Thus it may be concluded that the two sub-blocs are different and should not be united. Pollen fertility tends to be higher in sub-bloc $B$ than $A$, with general means of $44,6 \%$ and $40,07 \%$ of viable pollen respectively (see also general and partial means in quadro IX).

10 - Five of the 6 values of $t$ corresponding to the partial means of sub-blocs B (7th column in quadro IX) are relatively high, without being significant. Thus the appearance of one only possibly significant error "between rootstocks" is explained. On the other hand both extreme values of $\boldsymbol{t}$ for the partial means of sub-bloc $A$ are definitely significant. There can be no doubt that the orange "pera" on sour orange has significantly more viable pollen grains, while "limão cravo" reduces the viability. In absolute values, these effects are not very pronounced. They amount only to differences of $5 \%$ from the general mean.

11 - It is most remarkable that the 12 rootstocks behave rather differently in repetitions. The error between repetition and per rootstock are also given in quadro IX and there appear four highly significant values. Actually a grouping seems to be more reasonable, uniting the four significant values of $\sigma E R e p$ with a mean value of $\pm 9,60 \%$ and 11 degrees of freedom. The first value, when compared with the residual error of $\pm 4,51 \%$ is highly significant, while the latter is identical with it. One value has be left aside, being intermediate $( \pm 6,28 \%)$ thus permitting an inclusion in both groups.

The fact that for some rootstock, the plots turn out to be heterogeneous and for others not, may be atributed to small soil patches which are known to exist in the experimental plots at Limeira.

Further more it may be seem from quadro $\mathrm{X}$, that means per rootstock and repetition do not always show a parallel variation, thus explaining the appearance of a significant interaction in sub-bloc $B$.

\section{Grape-fruit “'Marsh-seedless"' Limeira}

12 - The results of this experiment seem much more complicated at first than those discussed above. All components per sub-bloc are significant, and the sub-blocs themselves are also different (first series of decompositions in quadro XI). But considering the means per rootstock (quadro XII) it is evident that the pollen fertility in Poncirus trifoliata is decidedly smaller than that in all other combinations. Thus, at first, Poncirus was excluded from the experiment (second series in 
quadro XI) and shall included again, but placed in contrast to the rest (least series in quadro XI). The difference between sub-blocs disappears now completely and we may accept the scheme of decomposition given in the lower right corner of the quadro XI as final. Here the three components: "between rootstocks", "between repetitions", and interaction are all significant and of the same magnitude.

13 - The genera! decomposition showed that Poncirus trifoliata occupies a special position. It causes a pronounced reduction in pollen fertility. For the remaining 11 rootstocks the analysis may be simplified since the two sub-blocs are not different anymore. The series of 11 partial means and the corresponding values of $\mathbf{t}$ form a continuous series with highly significant values at both extremes (quadro XII). Thus the effect of the rootstocks on pollen fertility of the scion is quite evident.

14 - As in the case of the "pera" orange, the different rootstock do not behave uniformly regarding to repetition. The individual values of $\sigma$ ERep in quadro XII (3rd and 4th columns from right) may be classified into two groups: one with small error, around a balanced mean of $\pm 10,05 \%$. The former is not significantly different from the residual error, while the latter differs significantly. In one instance, orange "azeda-doce", a classification seems difficult. It is interesting that the two mean errors are pratically the same as obtained before in the corresponding analysis for "pera", while individual rootstocks do not show an identical behaviour in both cases.

This seems to reinforce still more our assumption that soil patches, distribuited at randon, cause these differences of errors between repetitions.

15 - The significant interaction is caused by the irregular behaviour of the partial means of rootstock and repetition, given in quadro XIII.

16 - Since the percentage values obtained are relatively near the limit of $\mathrm{O}$, Fisher's angular transformation was applied (quadros XIV and XVI), but the results obtained did not offer any new results.

\section{Residual and theoretical errors}

17 - In order to analyse still further homogeneity of methods and to see if decomposition removes all systhematical variations, leaving variations due to chance only, comparison was made between residual error and theoretical error calculated by the formula : 


$$
\sigma \mathrm{i}= \pm \sqrt{\frac{\overline{\mathrm{v} \%(100-\overline{\mathrm{v}} \%)}}{\mathrm{n}}}
$$

where $\mathrm{n}$ stands for the mean number of pollen grains per slide counted; $\checkmark \%$ for the mean percentage of viable grains.

Generally speaking the residual errors are significantly larger than the theoretical values, while in one case $\vartheta$ is even significantly smaller than 1 . Considering that the number of grains counted per slide was very variable, we may be satisfied with the two corresponding values for the error (observed and ideal) which are always of about the same order.

\section{Results}

The results may be finally summarized as follows:

a) The method used was satisfatory.

b) The fertility of the pollen of the scion is to some extent influenced by the heterogeneity in the plots.

c) The rootstocks have definitely a pronounced effect upon pollen fertility of the scion.

\section{LITERATURA CITADA}

1. Cabral de Vasconcelos, P: W. Estudos comparativos da laranjeira Bá́a comum sobre 5 porta-enxertos. Bol. Secret. da Agric. Ind. e Com. Est. São Paulo. 1939.

2. Caryl, R. E. Citrus culture in California. California Agr. Ext. Service Circ. 114:1-48. 1940.

3. Clark, A. W. H. Leonard. The analysis of variance with special reference to data expressed as percentages. Jour. Amer. Soc. of Agronomy 31:55-66. 1936.

4. Gourley, J. H. e F. S. Howlett. Em Modern fruit production, 579 págs. The Macmillan Company. New York, 1941.

5. Haberlandt, G. Das Wesen der Crataegomespili. Sitzungsberichten der preussischen Akademie der Wissenschaften Phys-Math Klasse 20:1-23. 1930.

6. Haberlandt, G. Uber die Sonnen-und Schattenblater der Crataegomespili und und ihrer Eltern. Sitzungsberichten der preussischen Akademie der Wissenschaften Phys-Math Klasse 20:1-14.1934.

7. Haberlandt, G. Blattepidermis und Palisadengewebe der Crataegomespili und ihrer Eltern. Sitzungsberichten der preussischen Akademie der Wissenschaften Phys-Math Klasse 20:1-15. 1934.

8. Haberlandt, G. Beitrage zum Crataegomespili-Problem. Sitzungsberichten der preussischen Akademie der Wissenschaften Phys-Math Klasse 28:1-22. 1935.

9. Halma, F. F. Important consideration in selection of lemon scion varieties. The California Citrograph 22:493-506. 1937.

10. Hatton, R. G. The behaviour of certain pears on various guince rootstocks. Jour. of Pomology and Hort. Sci. 7:216-233. 1928. 
11. Hatton, R. G. Apple rootstocks studies. Effect of layered stocks upon the vigour and cropping of certain scions. Jour of Pomology and Hort. Sci. 13:293-350. 1935.

12. Hatton, R. G. Plum rootstocks studies : their effect on the vigour and cropping of the scion variety. Jour. of Pomology and Hort. Sci. 14:97-136. 1936.

13. Hodgson, R. W. outros. On the relation between vigour of scion variety and rootstock reaction in the lemon. The California Citrograph 23:290-306. 1938.

14. Hodgson, R. W. e outros. Rootstock and scion influence in citrus. The Cali fornia Citrograph 22:110. 1937.

15. Marloth, R. H. The citrus rootstock problem. Farming in South Africa 13:226-231. 1938.

16. Mendel, K. The anatomy and histology of the bud-union in citrus. The Palestine Jour. of Bot. and Hort. Sci. 1:13-46. 1936.

17. Mendel, $\mathbf{K}$. Some considerations on the anatomy and physiology of citrus budding. Hadar 10:60-64. 1937.

18. Moreira, S. Experiências de cavalos para citrus I. Bragantia 1: 525-565. Graph- 1-6. Fig. 1-20. 1941.

19. Moreira, S. e J. T. A. Gurgel. A fertilidade do polen $\theta$ sua correlação com o número de sementes, em espécies e formas do gênero Citrus. Bragantia 1: 669.711. 1941.

20. Nebel, B. R. Xenia and metaxenia in apples. New York Agr. Exp. St. Bul. 170:3-16. 1930.

21. Oppenheimer, H.P. The stock problem of the shamouti orange. Hadar 13:245-248 1940.

22. Ruggieri, G. Ricerche sull'affinitá d'innesto de limone "Monachello" com altri Citrus. Bol. della R. Stazione de Path. Vegetal. Firenze, Itália n.s. 16: 79-86, pl. 1-2. 1936.

23. Toxopeus, H. J. Stock-scion incompatibility in citrus and its cause. The Jour. of Pomology and Hort. Sci. 14:360-364. 1937.

24. Webber, H. J. Variations in citrus seedlings and their relation to rootstock selection. Hilgardia 7:1-79, 1932.

25. Yedidyah, S. Citrus varieties and rootstocks in Palestina. Hadar 11:39-40. 1938 


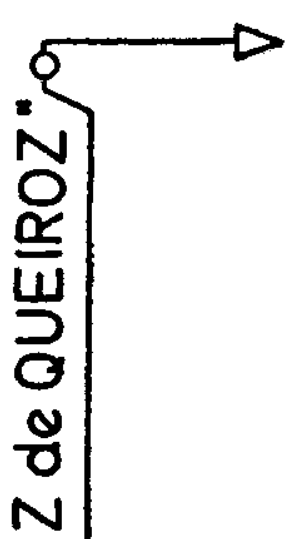

O $\infty N \infty \sim m N$ -

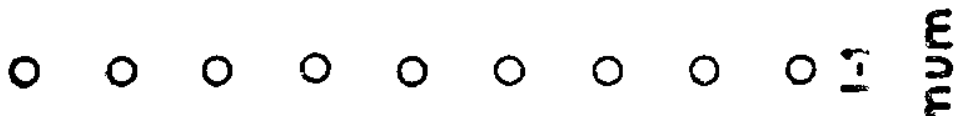

$\begin{array}{lllllllllll}0 & 0 & 0 & 0 & 0 & 0 & 0 & 0 & 0 & 0 & 0 \\ 0\end{array}$

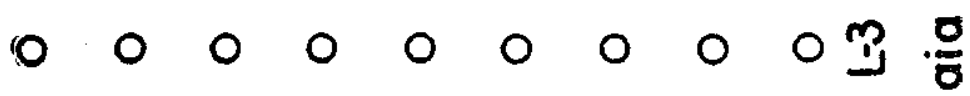

$\begin{array}{llllllllllll}0 & 0 & 0 & 0 & 0 & 0 & 0 & 0 & 0 & 7 & 1 \\ 0\end{array}$

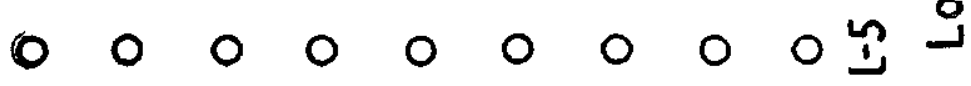

$\begin{array}{lllllllllll}0 & 0 & 0 & 0 & 0 & 0 & 0 & 0 & 0 & I_{0} & 0\end{array}$

$\frac{0}{0}$

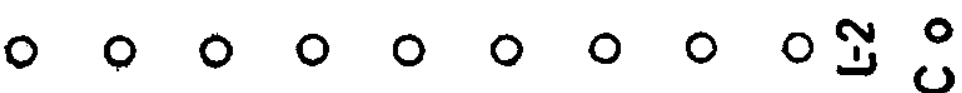

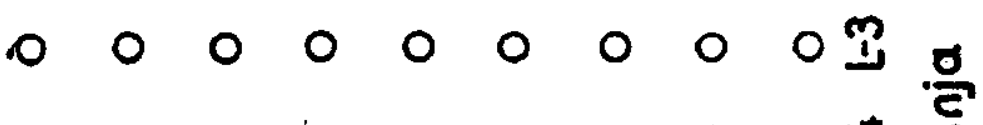

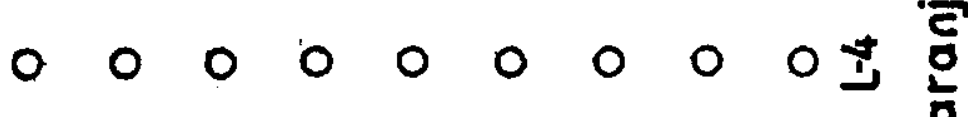

षृ

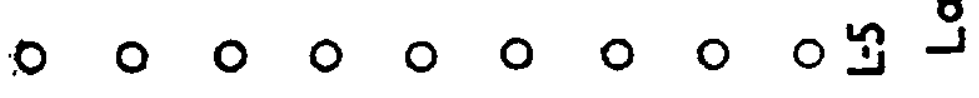

$\stackrel{0}{\geq}$

000000000000

ப。

용

임

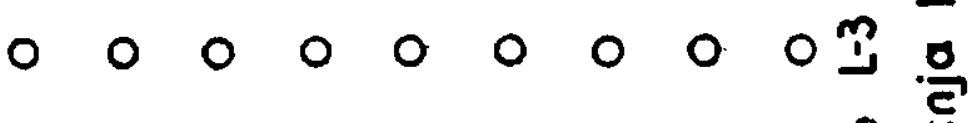

\begin{tabular}{lllllllllll}
0 & 0 & 0 & 0 & 0 & 0 & 0 & 0 & 0 & 7 & 0 \\
\hline
\end{tabular}

$\begin{array}{lllllllllll}0 & 0 & 0 & 0 & 0 & 0 & 0 & 0 & 0 & 3 & 7\end{array}$

它

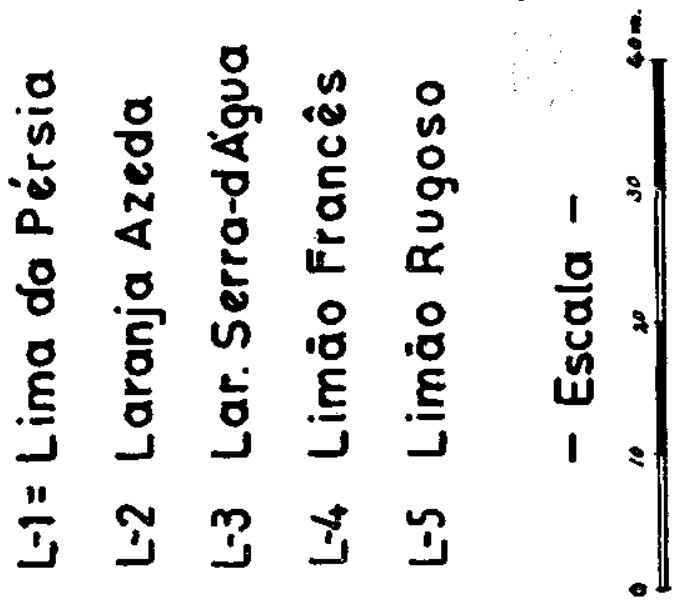




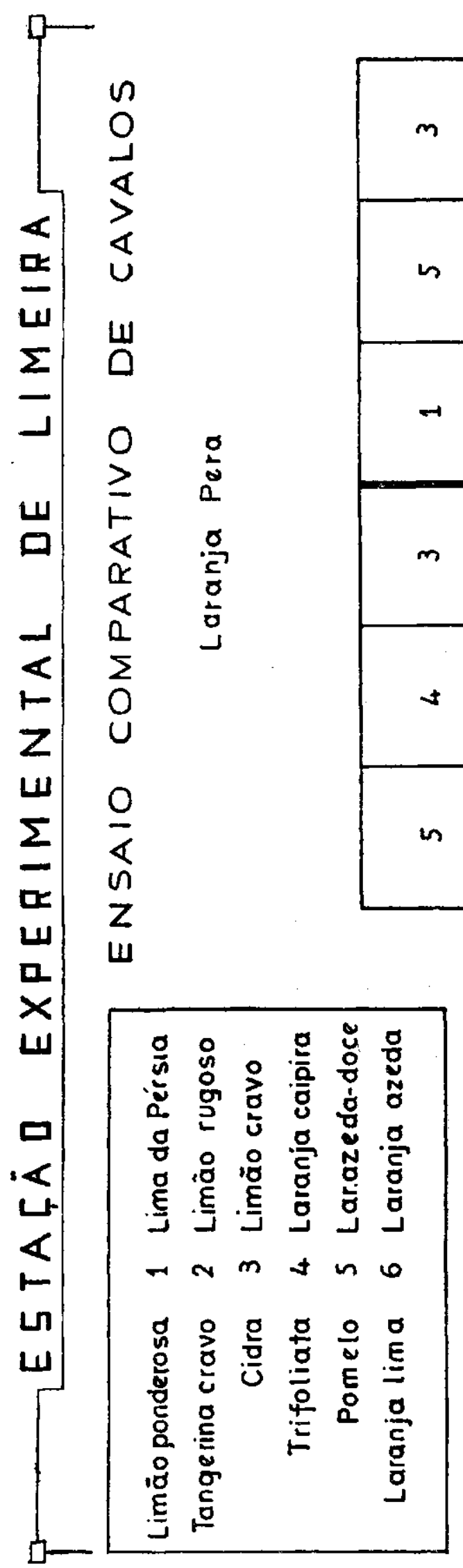

0
0
0
0
0
0
0
0
$\frac{0}{0}$
$\frac{0}{2}$ 


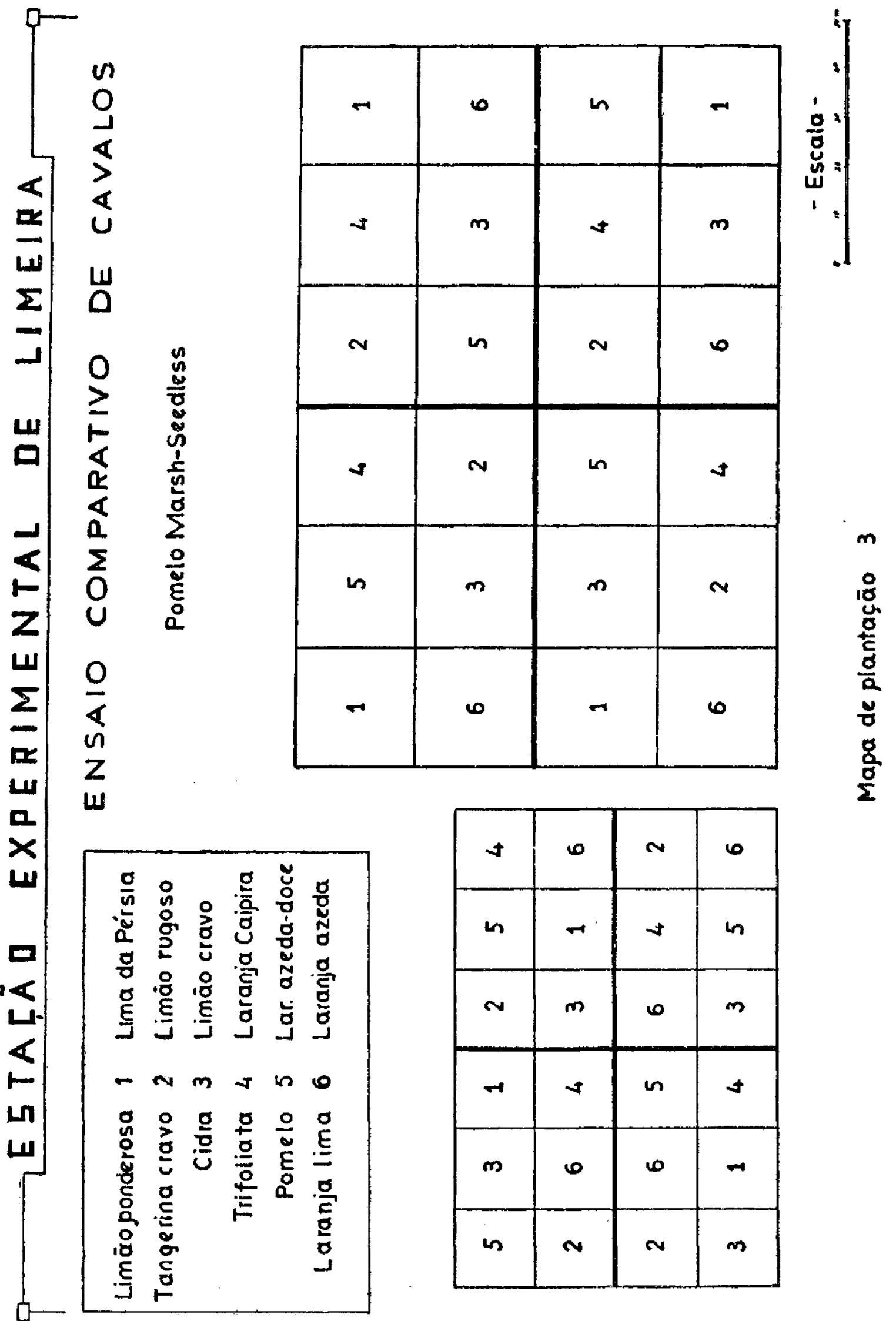

